Review article

\title{
The search for neuroimaging and cognitive endophenotypes: A critical systematic review of studies involving unaffected first-degree relatives of individuals with bipolar disorder
}

\author{
Kamilla W. Miskowiak ${ }^{\mathrm{a}, *, 1}$, Hanne L. Kjærstad ${ }^{\mathrm{a}, 1}$, Iselin Meluken ${ }^{\mathrm{a}}$, Jeff Zarp Petersen ${ }^{\mathrm{a}}$, \\ Beatriz R. Maciel $^{\text {b }}$, Cristiano A. Köhler ${ }^{b}$, Maj Vinberg ${ }^{\mathrm{a}}$, Lars V. Kessing ${ }^{\mathrm{a}}$, \\ André F. Carvalho ${ }^{b}$ \\ ${ }^{a}$ Copenhagen Affective Disorder Research Centre, Psychiatric Centre Copenhagen, Copenhagen University Hospital, Rigshospitalet, Copenhagen, Denmark \\ b Translational Psychiatry Research Group and Department of Clinical Medicine, Faculty of Medicine, Federal University of Ceará, Fortaleza, CE, Brazil
}

\section{A R T I C L E I N F O}

\section{Article history:}

Received 26 May 2016

Received in revised form 5 October 2016

Accepted 9 December 2016

Available online 12 December 2016

\section{Keywords:}

Bipolar disorder

Endophenotype

Cognition

Neurocognition

Neuroimaging

Psychiatry

Mood disorder

Pathophysiology

\begin{abstract}
A B S T R A C T
The phenomenology and underlying pathophysiology of bipolar disorder (BD) are heterogeneous. The identification of putative endophenotypes for $\mathrm{BD}$ can aid in the investigation of unique patho-etiological pathways, which may lead to the development of personalised preventative and therapeutic approaches for this multi-faceted disorder. We included original studies involving unaffected first-degree relatives of BD patients (URs) and a healthy control (HC) comparison group with no first-degree family history of mental disorders, investigating: 'cold' and 'hot' cognition and functional and structural neuroimaging. Seventy-seven cross-sectional studies met the inclusion criteria. The present review revealed that URs in comparison with HCs showed: (i) widespread deficits in verbal memory, sustained attention, and executive function; (ii) abnormalities in the reactivity to and regulation of emotional information along with aberrant reward processing, and heightened attentional interference by emotional stimuli; and (iii) less consistency in the findings regarding structural and resting state neuroimaging, and electrophysiological measures.
\end{abstract}

\section{Contents}

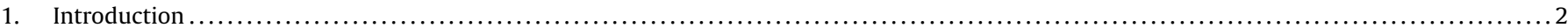

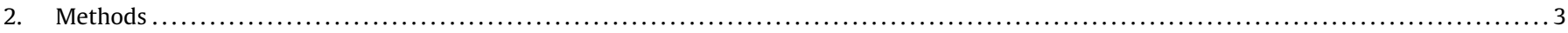

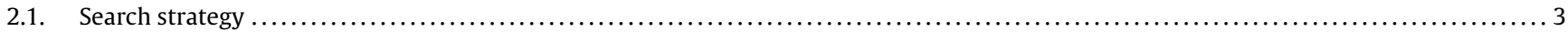

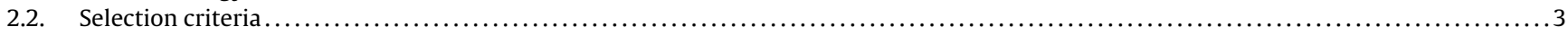

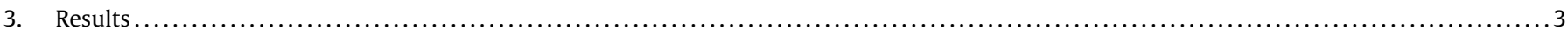

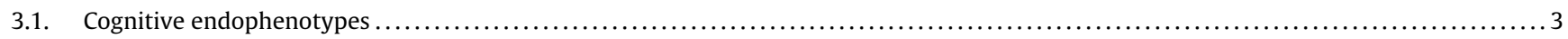

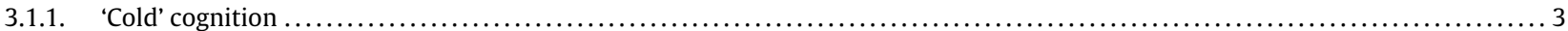

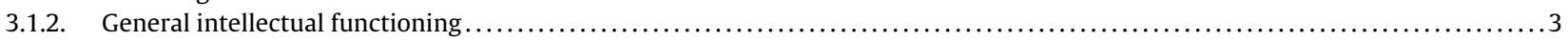

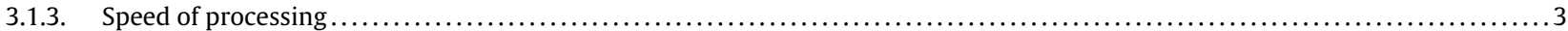

Abbreviations: ACC, anterior cingulate cortex; CPT, continuous performance task; dIPFC, dorsolateral prefrontal cortex; DSM, the Diagnostic and Statistical Manual of

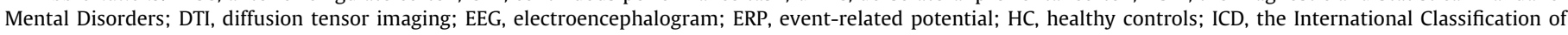

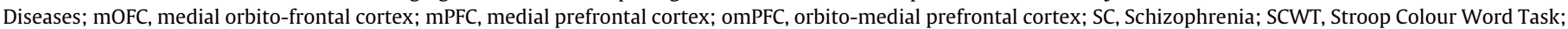

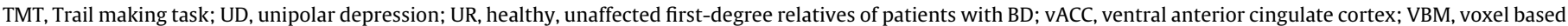

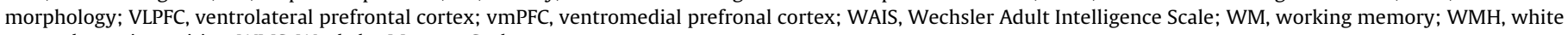
matter hyper intensities; WMS, Wechsler Memory Scale.

* Corresponding author at: Psychiatric Centre Copenhagen, Copenhagen University Hospital, Rigshospitalet, Blegdamsvej 9, DK-2100 Copenhagen, Denmark.

E-mail address: Kamilla@miskowiak.dk (K.W. Miskowiak).

1 Shared first authors. 


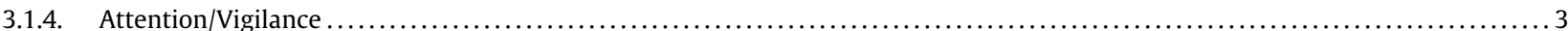

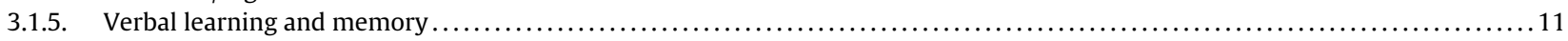

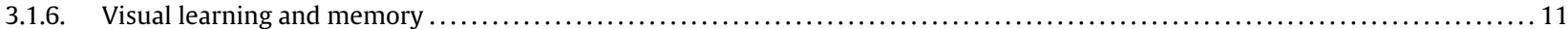

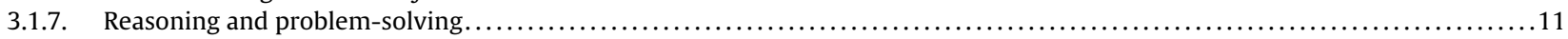

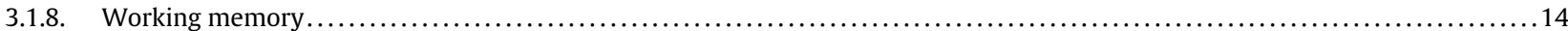

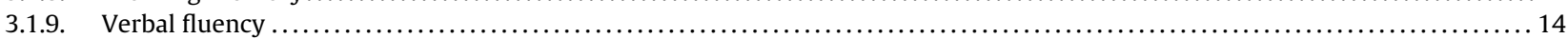

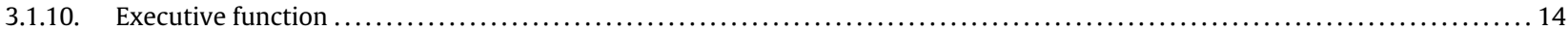

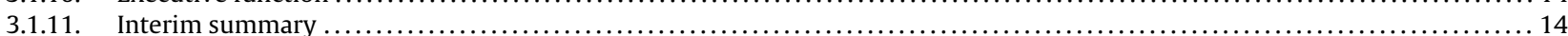

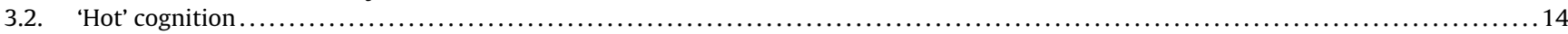

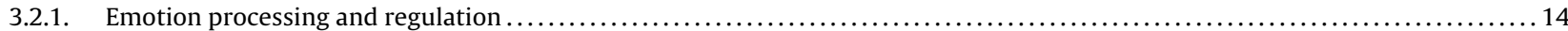

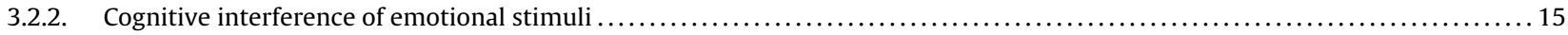

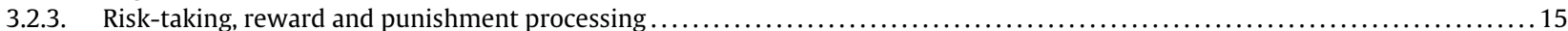

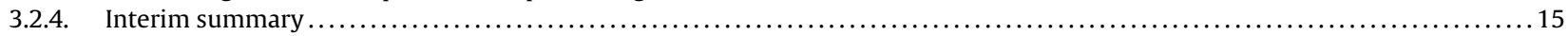

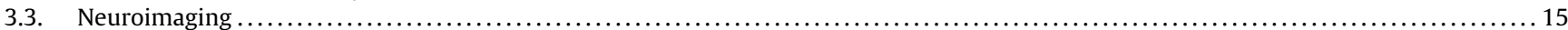

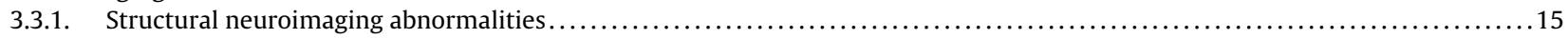

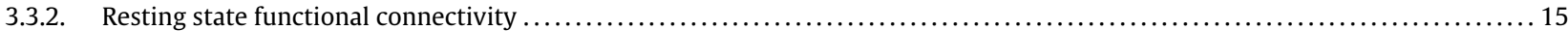

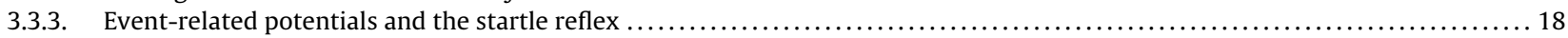

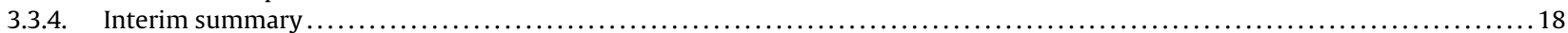

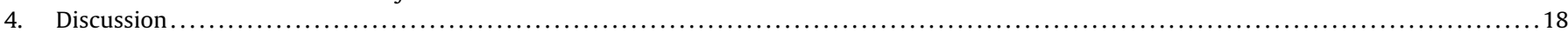

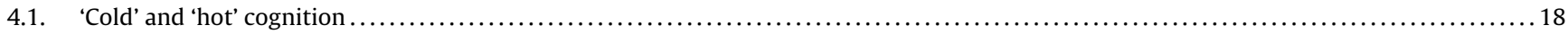

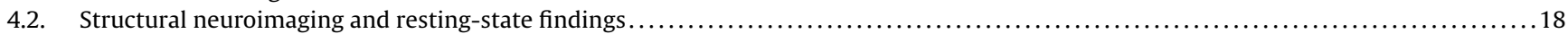

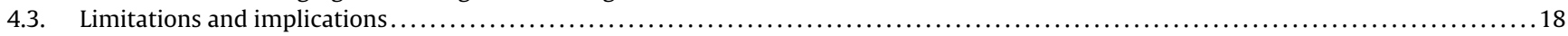

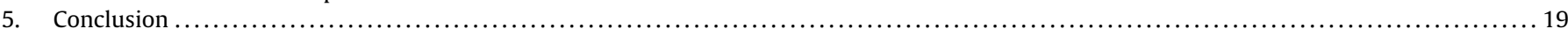

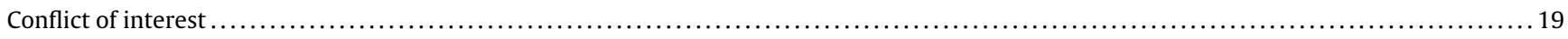

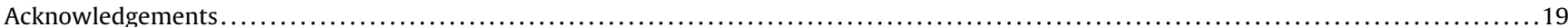

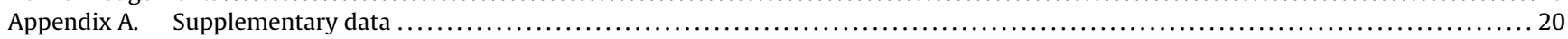

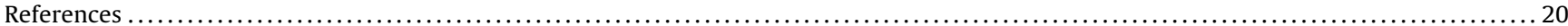

\section{Introduction}

Bipolar disorder (BD) is a common chronic illness that is characterised by extreme mood fluctuations and substantial cognitive impairment (Goodwin and Jamison, 2007; Grande et al., 2016). Although compelling evidence indicates that BD is associated with a high degree of heritability (e.g., Goes, 2016; Kieseppä et al., 2004; McGuffin et al., 2003), its exact pathophysiology remains elusive and involves a complex set of gene-environment interactions (Uher, 2014). Replication of genome wide association studies have proven to be difficult due to the complexity of the disorder, differences in diagnostic criteria, methodological challenges, and possible patho-etiological heterogeneity (Gatt et al., 2015; Kerner, 2015; McCarroll et al., 2014), although notably a recent study identified some putative biological pathways involved in the genetic predisposition to BD (e.g., hormonal regulation, calcium cannels; see Nurnberger et al., 2014). Endophenotypes are diseaseassociated traits that are highly heritable, associated with the illness, independent of the clinical state, and found in non-affected family members to a greater extent than in the general population (Gershon and Goldin, 1986; Gottesman and Gould, 2003; Leboyer et al., 1998). The past decades, the field has witnessed an intensive research effort in to putative endophenotypes for $\mathrm{BD}$, which may improve the understanding of disease heterogeneity through biological validation and phenotype stratification (Hasler et al., 2006; Kerner, 2015).

The search for candidate endophenotypes for BD has revealed substantial evidence for trait-related abnormalities across several neurocognitive domains (Balanza-Martinez et al., 2008) and neuroimaging measures (Hozer and Houenou, 2016; Wu et al., 2016). However, studies in unaffected relatives (URs) of BD patients have produced less uniform evidence for changes in neurocognitive function and neuroimaging measures. It is therefore crucial to evaluate which abnormalities are most consistently exhibited in genetically predisposed individuals to identify the most promising candidate endophenotypes for BD. These efforts may lead to identification of the most consistent biological pathways in BD.
Cognitive deficits are candidate endophenotypes of BD (e.g., Bora et al., 2009b; Glahn et al., 2010). These include disturbances in both 'cold' (i.e., non-emotional) and 'hot' (i.e., emotion-laden) cognition (Roiser and Sahakian, 2013). Trait-related deficits in 'cold' cognition have been repeatedly reported in individuals with BD across several neurocognitive domains, particularly within verbal memory and executive function (Bourne et al., 2013; Robinson et al., 2006; Torres et al., 2007), as well as in their unaffected relatives (Arts et al., 2008; Balanza-Martinez et al., 2008). Although changes in the processing of emotional information and emotional regulation are core abnormalities in mood disorders (Miskowiak and Carvalho, 2014; Phillips et al., 2008), these aspects of 'hot' cognition have only recently become a focus of scientific investigation in URs of patients with mood disorders. These studies suggest that emotion dysregulation is not only present in BD during acute mood episodes (Almeida and Phillips, 2013; Phillips et al., 2008) and in remission (Townsend et al., 2013), but also occur in genetically predisposed individuals (Heissler et al., 2014; Kanske et al., 2015).

Functional and structural imaging studies of BD have revealed fronto-limbic functional abnormalities (Chen et al., 2011a; Strakowski et al., 2012; Strakowski et al., 2005) coupled with structural changes, such as lateral ventricle enlargement (Arnone et al., 2009; Kempton et al., 2008; McDonald et al., 2004). However, little research has been conducted on URs of patients with BD (Mathias de Almeida et al., 2013). Functional neuroimaging studies of resting state activity in the prefrontal cortex, anterior cingulate cortex, and mesolimbic structures that subserve processing of emotionallyladen stimuli and emotion regulation show promise in revealing putative brain-based endophenotypes (Phillips and Vieta, 2007; Vargas et al., 2013). Indeed, aberrant neural response seems to be a more sensitive assay of abnormal brain function than overt behavioural or subjective measures (Haas et al., 2007). Nevertheless, the search for neuroimaging endophenotypes and a precise neuroimaging biosignature for BD has revealed discrepant findings (Phillips and Swartz, 2014) due to small and heterogeneous samples and different methodological approaches (Phillips and Kupfer, 2013). 
The aim of the present systematic review is to: (i) synthesise the extant findings in 'hot' and 'cold' cognition and structural and functional neuroimaging measures in unaffected first-degree relatives (URs), i.e., with no history of psychosis or mood disorder, of patients with in comparison with healthy controls (HCs); and in particular, (ii) to clarify which of the abnormalities within these domains constitute the most promising endophenotypes of $\mathrm{BD}$ that deserve further investigation in meta-analytical studies.

\section{Methods}

\subsection{Search strategy}

Computerised searches on the PubMed/MEDLINE, EMBASE, and PsychInfo databases were performed from inception up until April 2016 (see supplementary online material for the detailed search strategy used in this systematic review). Two reviewers independently performed title/abstract screening (kappa $=0.90$ ). Disagreements were discussed and consensus was reach in all cases. Full-texts of potentially eligible articles were retrieved and both reviewers considered these unique references for inclusion/exclusion during the secondary screening. The search strategy was augmented through tracking the citations of eligible articles in Google Scholar. This systematic review has followed the procedures of the Preferred reporting items for systematic reviews and meta-analyses (PRISMA) statement (Moher et al., 2009).

\subsection{Selection criteria}

We included original peer-reviewed articles involving: (a) first-degree unaffected relatives (URs) of individuals meeting Diagnostic and Statistical Manual of Mental Disorders (DSM) (Association, 2013) or International Classification of Diseases (ICD) (Organization, 1992) criteria for BD (including discordant twins) and a healthy control (HC) comparison group; (b) a diagnosis of BD established through a validated structured diagnostic interview; (c) investigated 'hot' or 'cold' cognition, functional or structural neuroimaging, and/or evoked potentials (ERP); (d) articles published in English, Portuguese, Spanish, Danish, German or French. We excluded articles that: (a) included samples with several diagnoses (unless data for BD was reported separately); (b) studies in which URs and HCs were not directly compared; (c) studies in which first-degree relatives and/or HCs had a history of psychosis, mood disorder, and/or substance use disorders to avoid confounding variables of interest; (d) genetic studies; and (e) meeting abstracts, reviews, and case reports. Whenever studies provided data from overlapping samples, we included only the report that included the largest dataset. In case of doubt, the corresponding author was contacted by e-mail. If reports with sample duplication investigated different variables of interest (i.e., within different overarching themes of interest and/or within different subdomains of cold cognition), both were included.

For studies on 'cold' cognition, we grouped the results of the individual neuropsychological tests into cognitive categories based on the Measurement and Treatment Research to Improve Cognition in Schizophrenia (MATRICS), except for verbal fluency which was separated from processing speed, according to the approach adopted by Bora and Pantelis (2015). The neuropsychological tests that were not part of the MATRICS were classified under the relevant cognitive domains based on factor loadings as reviewed by Rodriguez-Jimenez et al. (2012). Further, in accordance to the International Society for BD Task Force on neurocognition, we also included an 'executive function' domain to include response inhibition, cognitive flexibility, and attention switching (Van Rheenen and Rossell, 2014). Effect sizes were estimated whenever a given cognitive finding was clearly replicated across studies.

\section{Results}

After removal of duplicated hits, 1799 unique references were initially screened. After reviewing their title/abstracts, 493 fulltext articles were examined for eligibility. Of these 493 articles, 77 met inclusion criteria and were included in this review; 36 studies investigated 'cold' cognition alone, 21 studies assessed 'hot' cognition alone, 22 assessed neuroimaging alone, nine investigated 'cold' and 'hot' cognition, four investigated 'cold' cognition and neuroimaging, and two studies included all three areas of interest. Fig. 1 depicts the PRISMA flowchart. Given the amount of evidence in some of the aforementioned fields, when more than 10 studies are cited, we refer to the respective table for an overview of the data.

\subsection{Cognitive endophenotypes}

\subsection{1. 'Cold' cognition}

As seen from Table 1, 51 studies investigated 'cold' cognition (Adleman et al., 2014; Antila et al., 2007; Besnier et al., 2009; Bora et al., 2008; Brotman et al., 2014; Brotman et al., 2009; Brotman et al., 2008b; Chang and Lenzenweger, 2005; Christodoulou et al., 2012a,b; Civil Arslan et al., 2014; Costafreda et al., 2009; Daban et al., 2012; Deveci et al., 2013; Deveney et al., 2012; Dima et al., 2016; Doyle et al., 2009; Erk et al., 2014; Erol et al., 2014; Frangou, 2012; Frangou et al., 2005; Frantom et al., 2008; Hıdıroğlu et al., 2015; Juselius et al., 2009; Keri et al., 2001; Kim et al., 2015; Kim et al., 2012; Kosger et al., 2015; Kulkarni et al., 2010; Ladouceur et al., 2008; Li et al., 2015; Linke et al., 2013; Maziade et al., 2009; Nehra et al., 2014; Olsavsky et al., 2012; Patino et al., 2013; Pattanayak et al., 2012; Seidel et al., 2012; Sepede et al., 2012; Singh et al., 2014b; Sobczak et al., 2003; Sobczak et al., 2002; Szoke et al., 2006a,b; Teixeira et al., 2014; Thermenos et al., 2011; Trivedi et al., 2008; Tseng et al., 2015; Versace et al., 2010; Wessa et al., 2015; Zalla et al., 2004); these examined general intellectual functioning $(\mathrm{k}=31 ; \mathrm{N}=844 \mathrm{URs}, 1040 \mathrm{HCs})$, speed of processing $(\mathrm{k}=14 ; \mathrm{N}=630$ URs, $551 \mathrm{HCs}$ ), attention/vigilance ( $\mathrm{k}=13 ; \mathrm{N}=408$ URs, $396 \mathrm{HCs}$ ), verbal learning and memory $(\mathrm{k}=18 ; \mathrm{N}=654 \mathrm{URs}, 635 \mathrm{HCs})$, visual learning and memory ( $\mathrm{k}=9 ; \mathrm{N}=341 \mathrm{URs}, 389 \mathrm{HCs})$, reasoning and problem solving $(\mathrm{k}=20 ; \mathrm{N}=718 \mathrm{URs}, 734 \mathrm{HCs})$, working memory $(\mathrm{k}=12 ; \mathrm{N}=390 \mathrm{URs}, 412 \mathrm{HCs})$, verbal fluency ( $\mathrm{k}=12 ; \mathrm{N}=307 \mathrm{URs}$, $440 \mathrm{HCs}$ ), and executive function ( $\mathrm{k}=25 ; \mathrm{N}=888 \mathrm{URs}, 981 \mathrm{HCs}$ ) (for an overview and study details see Table 1 ).

\subsubsection{General intellectual functioning}

All but one small study (Frantom et al., 2008) found no difference between URs and HCs on IQ measures, which indicates that general intelligence is not an illness-trait conferring genetic liability for BD.

\subsubsection{Speed of processing}

Nine of 11 studies using the Trail Making Test A(TMT-A) showed no reduction in speed of processing in URs (Table 1 ). A recent study found that only URs with two or more relatives with BD displayed poorer psychomotor speed, suggesting a possible influence of genetic load on this cognitive domain (Kosger et al., 2015). In contrast, two of four (i.e., half) studies using the WAIS digit symbol subtest revealed slower performance in URs (Antila et al., 2007; Daban et al., 2012), possibly executive function and memory elements in this test. However, overall these finding provide little indication for slower psychomotor speed in URs.

\subsubsection{Attention/Vigilance}

Of studies investigating sustained attention $(\mathrm{k}=13)$, half found impaired performance in URs (Adleman et al., 2014; Brotman et al., 
Table

Eligible studies $(\mathrm{k}=51)$ in which different domains related to 'cold' cognition were compared between unaffected relatives and healthy controls.

\begin{tabular}{|c|c|c|c|c|c|c|c|}
\hline Author & Paradigm & Measure & $N$ & Age & Gender (\% female) & UR family status & Finding \\
\hline \multicolumn{8}{|l|}{ Intelligence } \\
\hline Antila et al. (2007) & $\begin{array}{l}\text { WAIS-R: Vocabulary } \\
\text { subtest }\end{array}$ & Behaviour & 40 URs, 55 HCs & $51.4(9.1)$ & 53 & Mixed & $\mathrm{URs}=\mathrm{HCs}$ \\
\hline Bora et al. (2008) & $\begin{array}{l}\text { WAIS-R: Information } \\
\text { subtest }\end{array}$ & Behaviour & 34 URs, 25 HCs & $45.7(12.2)$ & 59 & Siblings, parents & $\mathrm{URs}=\mathrm{HCs}$ \\
\hline Brotman et al. (2008b) & WASI- two scale IQ & Behaviour & 25 URs, 36 HCs & $12.15(3.05)$ & 28 & Offspring, siblings & $\mathrm{URs}=\mathrm{HCs}$ \\
\hline Brotman et al. (2009) & WASI-FSIQ & Behaviour & 26 URs, 24 HCs & $12.0(3.0)$ & 31 & Offspring, siblings & $\mathrm{URs}=\mathrm{HCs}$ \\
\hline Brotman et al. (2014) & WASI- FSIQ & Behaviour & 15 URs, 29 HCs & $14.5(2.2)$ & 40 & Offspring, siblings & $\mathrm{URs}=\mathrm{HCs}$ \\
\hline Christodoulou et al. $(2012 \mathrm{a}, \mathrm{b})^{\mathrm{a}}$ & $\begin{array}{l}\text { WAIS-R: Vocabulary } \\
\text { Subtest }\end{array}$ & Behaviour & 17 URs, 23 HCs & $38.7(13.4)$ & 76 & Mixed & $\mathrm{URs}=\mathrm{HCs}$ \\
\hline Costafreda et al. (2009) & WASI verbal IQ & fMRI & 7 URs, 48 HCs & $39.4(15.8)$ & 86 & Co-twins & $\mathrm{URs}=\mathrm{HCs}$ \\
\hline Daban et al. (2012) & $\begin{array}{l}\text { WAIS-III: Vocabulary } \\
\text { sub-test }\end{array}$ & Behaviour & 50 URs, 60 HCs & $39.3(13.7)$ & 67 & - & $\mathrm{URs}=\mathrm{HCs}$ \\
\hline Deveney et al. (2012) & WASI two-scale IQ & Behaviour, fMRI & 13 URs, $21 \mathrm{HC}$ & $13.5(1.8)$ & 54 & Offspring, siblings & $\mathrm{URs}=\mathrm{HCs}$ \\
\hline Doyle et al. (2009) & $\begin{array}{l}\text { WISC-III or WAIS-III: } \\
\text { Vocabulary and block } \\
\text { design subtest }\end{array}$ & Behaviour & 118 URs, 79 HCs & $12.8(4.0)$ & 47 & Siblings & $\mathrm{URs}=\mathrm{HCs}$ \\
\hline Erk et al. (2014) & $\begin{array}{l}\text { Mehrfachwahl- } \\
\text { Wortschatz- } \\
\text { Intelligenztes (verbal } \\
\text { IQ) }\end{array}$ & Behaviour, fMRI & 59 URs, $110 \mathrm{HCs}$ & $31.8(11.8)$ & 61 & Mixed & $\mathrm{URs}=\mathrm{HCs}$ \\
\hline Frangou et al. (2005) & WAIS-R FSIQ & Behaviour & 15 URs, 43 HCs & $27.2(8.9)$ & 67 & Offspring & $\mathrm{URs}=\mathrm{HCs}$ \\
\hline Frangou (2012) & WAIS-R FSIQ & Behaviour, fMRI & 48 URs 71 HCs & $36.5(13.8)$ & 52 & Siblings, offspring & $\mathrm{URs}=\mathrm{HCs}$ \\
\hline Frantom et al. (2008) & $\begin{array}{l}\text { WAIS III: Vocabulary } \\
\text { and block design } \\
\text { subtests }\end{array}$ & Behaviour & 19 URs, 19 HCs & $38.3(15.7)$ & 79 & Mixed & $\mathrm{URs}<\mathrm{HCs}$ \\
\hline Keri et al. (2001) & WAIS-R FSIQ & Behaviour & 20 URs, 20 HCs & $35.1(9.5)$ & 60 & Siblings & $\mathrm{URs}=\mathrm{HCs}$ \\
\hline Kim et al. (2015) & $\begin{array}{l}\text { Korean WAIS: } \\
\text { Vocabulary and block } \\
\text { design }\end{array}$ & Behaviour & 29 URs, 34 HCs & $31.8(8.0)$ & 59 & - & $\mathrm{URs}=\mathrm{HCs}$ \\
\hline Ladouceur et al. (2008) & WISC-III: FSIQ & Behaviour & 20 URs, 22 HCs & $13.0(2.7)$ & 55 & Offspring & $\mathrm{URs}=\mathrm{HCs}$ \\
\hline Linke et al. (2013) & $\begin{array}{l}\text { Multiple Choice Word } \\
\text { Vocabulary (IQ) }\end{array}$ & Behaviour & 22 URs, 22 HCs & $28.0(11.0)$ & 50 & Siblings, offspring & $\mathrm{URs}=\mathrm{HCs}$ \\
\hline Maziade et al. (2009) & $\begin{array}{l}\text { WISC-III or WAIS-III for } \\
\text { relatives and WASI for } \\
\text { relatives }\end{array}$ & Behaviour & 23 URs, 45 HCs & $17.5(4.5)$ & 39 & Offspring & URs $<$ HCs \\
\hline Olsavsky et al. (2012) & WASI FSIQ & Behaviour & 13 URs, 56 HCs & $14.0(2.4)$ & 46 & Offspring, siblings & $\mathrm{URs}=\mathrm{HCs}$ \\
\hline Seidel et al. (2012) & $\begin{array}{l}\text { Mehrfachwahl- } \\
\text { Wortschatz- } \\
\text { Intelligenztes (verbal } \\
\text { IQ) }\end{array}$ & Behaviour & 21 URs, 21 HCs & $38.4(17.7)$ & 48 & - & $\mathrm{URs}=\mathrm{HCs}$ \\
\hline Sepede et al. (2012) & WAIS FSIQ & Behaviour, fMRI & 22 URs, 24 HCs & $31.5(7.3)$ & 68 & Offspring, siblings & $\mathrm{URs}=\mathrm{HCs}$ \\
\hline Singh et al. (2014b) & WASI FSIQ & Behaviour & 20 URs, 25 HCs & $12.7(2.9)$ & 65 & Offspring & $\mathrm{URs}=\mathrm{HCs}$ \\
\hline Sobczak et al. (2002) & $\begin{array}{l}\text { Groninger Intelligence } \\
\text { Test (GIT) }\end{array}$ & Behaviour & 30 URs, 15 HCs & $41.4(2.6)$ & 73 & Mixed & $\mathrm{URs}=\mathrm{HCs}$ \\
\hline Sobczak et al. (2003) & $\begin{array}{l}\text { Groninger Intelligence } \\
\text { Test (GIT) }\end{array}$ & Behaviour & 30 URs, 15 HCs & $41.0(2.9)$ & 67 & Mixed & $\mathrm{URs}=\mathrm{HCs}$ \\
\hline Teixeira et al. (2014) & $\begin{array}{l}\text { WASI (no info if full } \\
\text { scale or not) }\end{array}$ & Behaviour & 18 URs, 20 HCs & $12.7(3.1)$ & 50 & Offspring & $\mathrm{URs}=\mathrm{HCs}$ \\
\hline Thermenos et al. (2011) & $\begin{array}{l}\text { WAIS or WISC } \\
\text { (Estimated IQ with } \\
\text { eight subtest) }\end{array}$ & Behaviour, fMRI & $10 \mathrm{URs}, 10 \mathrm{HCs}$ & $18.4(4.2)$ & 50 & - & $\mathrm{URs}=\mathrm{HCs}$ \\
\hline Versace et al. (2010) & WASI FSIQ & Behaviour & 20 URs, 25 HCs & $13.2(2.5)$ & 45 & Offspring & $\mathrm{URs}=\mathrm{HCs}$ \\
\hline
\end{tabular}




\begin{tabular}{|c|c|c|c|c|c|c|c|}
\hline Wessa et al. (2015) & $\begin{array}{l}\text { German Culture Fair } \\
\text { Intelligence test }\end{array}$ & Behaviour & 27 URs, 29 HCs & $31.8(14.4)$ & 52 & - & $\mathrm{URs}=\mathrm{HCs}$ \\
\hline Zalla et al. (2004) & WAIS-R FSIQ & Behaviour & 33 URs, 20 HCs & $37.3(11.0)$ & 61 & Parents, siblings & $\mathrm{URs}=\mathrm{HCs}$ \\
\hline \multicolumn{8}{|l|}{ Speed of processing } \\
\hline Antila et al. (2007) & TMT A, Digit Symbol & Behaviour & 40 URs, 55 HCs & $51.4(9.1)$ & 53 & Mixed & $\begin{array}{l}\text { Digit Symbol: UR }<\text { HC; TMT } \\
\text { A: UR = HC }\end{array}$ \\
\hline Arslan et al. (2014) & TMT A & Behaviour & 55 URs, $32 \mathrm{HCs}$ & $37.7(3.6)$ & 56 & Mixed & $\mathrm{URs}=\mathrm{HCs}$ \\
\hline Bora et al. (2008) & TMT A & Behaviour & 34 URs, 25 HCs & $45.7(12.2)$ & 59 & Siblings, parents & $\mathrm{URs}=\mathrm{HCs}$ \\
\hline Daban et al. (2012) & Digit symbol & Behaviour & $50 \mathrm{URs}, 60 \mathrm{HCs}$ & $39.3(13.7)$ & 67 & - & $\mathrm{URs}<\mathrm{HCs}$ \\
\hline Deveci et al. (2013) & TMT A & Behaviour & 30 URs, 37 HCs & $12.3(2.8)$ & 50 & Offspring & URs $<$ HCs \\
\hline Doyle et al. (2009) & $\begin{array}{l}\text { Digit Symbol Coding, } \\
\text { Digit Symbol Search }\end{array}$ & Behaviour & 118 URs, 79 HCs & $12.8(4.0)$ & 47 & Siblings & $\begin{array}{l}\text { Digit Symbol Coding: } \\
\text { URs = HCs Digit Symbol } \\
\text { Search: URs = HCs }\end{array}$ \\
\hline Erol et al. (2014) & TMT A & Behaviour & 50 URs, 50 HCs & $56.2(6.7)$ & 50 & Parents & $\mathrm{URs}=\mathrm{HCs}$ \\
\hline Frantom et al. (2008) & TMT A & Behaviour & 19 URs, 19 HCs & $38.3(15.7)$ & 79 & Mixed & $\mathrm{URs}=\mathrm{HCs}$ \\
\hline Kosger et al. (2015) & TMT A & Behaviour & $\begin{array}{l}24 \mathrm{FP}(\geq \text { two relatives } \\
\text { with } \mathrm{BD}), 26 \mathrm{SP} \text { (One } \\
\text { first-degree relative), } \\
26 \mathrm{HCs}\end{array}$ & $\begin{array}{l}\text { FP: } 57.5 \text { (7.6); SP: } 56.1 \\
(5.8)\end{array}$ & FP: 46; SP: 54 & Mixed & $\mathrm{FPs}<\mathrm{HCs} ; \mathrm{SPs}=\mathrm{HCs}$ \\
\hline Kulkarni et al. (2010) & TMT A & Behaviour & 30 URs, $30 \mathrm{HCs}$ & $28.9(7.1)$ & 20 & Siblings & $\mathrm{URs}=\mathrm{HCs}$ \\
\hline Nehra et al. (2014) & Digit substitution & Behaviour & 20 URs, 20 HCs & $36.9(8.6)$ & 15 & Siblings & $\mathrm{URs}=\mathrm{HCs}$ \\
\hline Pattanayak et al. (2012) & TMT A & Behaviour & 20 URs, 20 HCs & 30.7 (11.4) & 40 & Mixed & $\mathrm{URs}=\mathrm{HCS}$ \\
\hline Szoke et al. (2006b) & TMT A & Behaviour & 51 URs, $50 \mathrm{HCs}$ & $40.4(13.3)$ & 63 & - & $\mathrm{URs}=\mathrm{HCs}$ \\
\hline Szoke et al. (2006a) & TMT A & Behaviour & 63 URs, 48 HCs & $40.7(13.0)$ & 61 & - & $\mathrm{URs}=\mathrm{HCs}$ \\
\hline \multicolumn{8}{|l|}{ Attention/vigilance } \\
\hline Adleman et al. (2014) & The Flanker task & Behaviour & 15 URs, 34 HCs & $4.6(0.6)$ & 27 & Offspring, siblings & $\begin{array}{l}\text { URs }<\text { HCs (variability of RT } \\
\text { on incongruent trails) }\end{array}$ \\
\hline Bora et al. (2008) & Conners' CPT-II & Behaviour & 34 URs, 25 HCs & $45.7(12.2)$ & 59 & Siblings, parents & $\mathrm{URs}=\mathrm{HCs}$ \\
\hline Brotman et al. (2009) & Flanker CPT & Behaviour & 26 URs, 24 HCs & $12.0(3.0)$ & 31 & Offspring, siblings & $\mathrm{URs}<\mathrm{HC}$ \\
\hline Deveci et al. (2013) & $\begin{array}{l}\text { Test of variables of } \\
\text { attention (TOVA) }\end{array}$ & Behaviour & 30 URs, 37 HCs & $12.3(2.8)$ & 50 & Offspring & URs $=\mathrm{HCs}$ \\
\hline Doyle et al. (2009) & Seidman auditory CPT & Behaviour & 118 URs, 79 HCs & $12.8(4.0)$ & 47 & Siblings & $\mathrm{URs}<\mathrm{HCs}$ \\
\hline Frantom et al. (2008) & CPT & Behaviour & 19 URs, 19 HCs & $38.3(15.7)$ & 79 & Mixed & $\mathrm{URs}=\mathrm{HCs}$ \\
\hline Kim et al. (2015) & $\begin{array}{l}\text { Auditory and visual } \\
\text { CPT }\end{array}$ & Behaviour & 29 URs, $34 \mathrm{HCs}$ & $31.8(8.0)$ & 59 & - & $\mathrm{URs}=\mathrm{HCs}$ \\
\hline Kulkarni et al. (2010) & СРT & Behaviour & 30 URs, 30 HCs & $28.9(7.1)$ & 20 & Siblings & $\mathrm{URs}=\mathrm{HCs}$ \\
\hline Maziade et al. (2009) & CPT-II & Behaviour & 23 URs, 45 HCs & $17.5(4.5)$ & 39 & Offspring & $\mathrm{URs}=\mathrm{HCs}$ \\
\hline Patino et al. (2013) & $\begin{array}{l}\text { The Eriksen Flanker } \\
\text { Task }\end{array}$ & Behaviour & 22 URs, 20 HCs & $15.0(3.0)$ & 46 & Offspring & URs $<$ HCs \\
\hline Sepede et al. (2012) & Degraded stimulus CPT & Behaviour, fMRI & 22 URs, 24 HCs & $31.5(7.3)$ & 68 & Offspring, siblings & $\begin{array}{l}\text { Behaviour: URs }<\text { HCs. } \\
\uparrow \text { activation in bilateral } \\
\text { insula and posterior } \\
\text { middle cingulate cortex } \\
\text { during non-correct target } \\
\text { response. During correct } \\
\text { target response, URs } \\
\text { showed } \uparrow \text { deactivation of } \\
\text { posterior cingulate, and } \\
\text { only during stimuli } \\
\text { degradation } \uparrow \text { activity in } \\
\text { left insula and bilateral } \\
\text { inferior parietal lobule. }\end{array}$ \\
\hline
\end{tabular}


Table 1 (Continued)

\begin{tabular}{|c|c|c|c|c|c|c|c|}
\hline Author & Paradigm & Measure & $N$ & Age & Gender (\% female) & UR family status & Finding \\
\hline Sobczak et al. (2003) & $\begin{array}{l}\text { Dichotic Listening task } \\
\text { (DLT), Left/right Choice } \\
\text { reaction time (CRT), } \\
\text { Motor choice reaction } \\
\text { time (MRCT) }\end{array}$ & Behaviour & 30 URs, 15 HCs & $41.0(2.9)$ & 67 & Mixed & $\begin{array}{l}\text { DLT: URs }<\text { HCs, MCR; } \\
\text { Us<HCs, CRT URs = HCS. (FH } \\
\text { I vs. II: DLT: URs = HCs, } \\
\text { MRCT: URs }<\text { HCs, CRT } \\
\text { URs = HCs. Group x TRP } \\
\text { loading interaction: DLT: } \\
\text { URs = HC, MCRT: URs = HCS, } \\
\text { CRT: URs }>\text { HCs) }\end{array}$ \\
\hline Trivedi et al. (2008) & СРТ & Behaviour & 10 URs, $10 \mathrm{HCs}$ & $30.1(11.2)$ & 10 & Siblings & URs $<$ HCs \\
\hline \multicolumn{8}{|l|}{ Verbal learning and memory } \\
\hline Antila et al. (2007) & CVLT & Behaviour & 40 URs, 55 HCs & $51.4(9.1)$ & 53 & Mixed & $\mathrm{URs}=\mathrm{HCs}$ \\
\hline Arslan et al. (2014) & RAVLT & Behaviour & 55 URs, $32 \mathrm{HCs}$ & $37.7(3.6)$ & 56 & Mixed & $\mathrm{URs}<\mathrm{HCs}$ \\
\hline Bora et al. (2008) & RAVLT & Behaviour & 34 URs, 25 HCs & $45.7(12.2)$ & 59 & Siblings, parents & $\mathrm{URs}=\mathrm{HCs}$ \\
\hline Chang and Lenzenweger (2005) & $\begin{array}{l}\text { The Miller-Selfridge } \\
\text { Task }\end{array}$ & Behaviour & 30 URs, 30 HCs & - & 47 & - & $\mathrm{URs}=\mathrm{HCs}$ \\
\hline Christodoulou et al. (2012a) & CVLT & Behaviour & 17 URs, 23 HCs & $38.7(13.4)$ & 76 & Mixed & URs $<$ HCs \\
\hline Deveci et al. (2013) & RAVLT & Behaviour & 30 URs, 37 HCs & $12.3(2.8)$ & 50 & Offspring & $\mathrm{URs}<\mathrm{HCs}$ \\
\hline Doyle et al. (2009) & $\begin{array}{l}\text { CVLT-II (trails 1-5 } \\
\text { only) }\end{array}$ & Behaviour & 118 URs, 79 HCs & $12.8(4.0)$ & 47 & Siblings & URs $=\mathrm{HCs}$ \\
\hline Erk et al. (2014) & $\begin{array}{l}\text { Verbal learning and } \\
\text { memory task (VLMT) }\end{array}$ & Behaviour & 59 URs, $110 \mathrm{HCs}$ & $31.8(11.8)$ & 61 & Mixed & $\mathrm{URs}=\mathrm{HCs}$ \\
\hline Frantom et al. (2008) & CVLT & Behaviour & 19 URs, 19 HCs & $38.3(15.7)$ & 79 & Mixed & $\mathrm{URs}=\mathrm{HCs}$ \\
\hline Keri et al. (2001) & $\begin{array}{l}\text { Verbal recall and } \\
\text { recognition }\end{array}$ & Behaviour & 20 URs, 20 HCs & $35.1(9.5)$ & 60 & Siblings & URs $<\mathrm{HCs}$ \\
\hline Kim et al. (2015) & K-AVLT & Behaviour & 29 URs, 34 HCs & $31.8(8.0)$ & 59 & - & URs $=\mathrm{HCs}$ \\
\hline Kosger et al. (2015) & CVLT & Behaviour & $\begin{array}{l}24 \mathrm{FP} \text { ( } \geq \text { two relatives } \\
\text { with } \mathrm{BD}), 26 \mathrm{SP} \text { (One } \\
\text { first-degree relative), } \\
26 \mathrm{HCs}\end{array}$ & $\begin{array}{l}\text { FP: } 57.5 \text { (7.6); SP: } 56.1 \\
(5.8)\end{array}$ & FP: 46; SP: 54 & Mixed & $\mathrm{FD}<\mathrm{HCs}, \mathrm{SP}=\mathrm{HCs}$ \\
\hline Kulkarni et al. (2010) & RAVLT & Behaviour & $30 \mathrm{URs}, 30 \mathrm{HCs}$ & $28.9(7.1)$ & 20 & Siblings & $\mathrm{URs}<\mathrm{HCs}$ \\
\hline Maziade et al. (2009) & CVLT & Behaviour & 23 URs, 45 HCs & $17.5(4.5)$ & 39 & Offspring & $\mathrm{URs}<\mathrm{HCs}$ \\
\hline Nehra et al. (2014) & $\begin{array}{l}\text { Hopkins verbal } \\
\text { learning test-revised } \\
\text { (HVLT-R) }\end{array}$ & Behaviour & 20 URs, 20 HCs & $36.9(8.6)$ & 15 & Siblings & URs $<$ HCs \\
\hline Pattanayak et al. (2012) & PGI Memory Scale & Behaviour & 20 URs, 20 HCs & $30.7(11.4)$ & 40 & Mixed & $\mathrm{URs}=\mathrm{HCs}$ \\
\hline Sobczak et al. (2002) & $\begin{array}{l}\text { Visual verbal learning } \\
\text { task (VVLT) }\end{array}$ & Behaviour & 30 URs, 15 HCs & $41.4(2.6)$ & 73 & Mixed & $\begin{array}{l}\text { URs }<\text { HCs. URs(BD } \\
\text { I) }<\text { URs(BDII). Group x trp } \\
\text { depletion effects: } \\
\text { URs = HCs }\end{array}$ \\
\hline Sobczak et al. (2003) & $\begin{array}{l}\text { Visual verbal learning } \\
\text { task (VVLT) }\end{array}$ & Behaviour & 30 URs, 15 HCs & $41.0(2.9)$ & 67 & Mixed & $\begin{array}{l}\text { URs }<\text { HCs. } \\
\text { URs(BDI) }<\text { URs(BDII). } \\
\text { Group x trp loading } \\
\text { interaction: URs = URs }\end{array}$ \\
\hline $\begin{array}{l}\text { Visual learning and memory } \\
\text { Doyle et al. (2009) }\end{array}$ & $\begin{array}{l}\text { Rey-Osterreich } \\
\text { Complex figure }\end{array}$ & Behaviour & 118 URs, 79 HCs & $12.8(4.0)$ & 47 & Siblings & URs $=\mathrm{HCs}$ \\
\hline Erk et al. (2014) & & fMRI & 59 URs $110 \mathrm{HCs}$ & $31.8(11.8)$ & 61 & Mixed & $\begin{array}{l}\downarrow \text { activity in left and right } \\
\text { hippocampus and pgACC }\end{array}$ \\
\hline
\end{tabular}




\begin{tabular}{|c|c|c|c|c|c|c|c|}
\hline Frantom et al. (2008) & $\begin{array}{l}\text { Rey-Osterrieth } \\
\text { Complex Figure (ROCF), } \\
\text { Biber Figure Learning } \\
\text { Test-Extended (Biber), } \\
\text { Faces I and II subtests } \\
\text { of the Wechsler } \\
\text { Memory Scale III, } \\
\text { Benton Facial } \\
\text { Recognition }\end{array}$ & Behaviour & 19 URs, 19 HCs & $38.3(15.7)$ & 79 & Mixed & $\begin{array}{l}\text { Biber } 1-5 \text { trail: URs }<\text { HCs, } \\
\text { ROCF:URs }<\text { HCs, } \downarrow \\
\text { performance on WMS } \\
\text { Faces I. No other group } \\
\text { differences. }\end{array}$ \\
\hline Kim et al. (2015) & K-Complex figure task & Behaviour & 29 URs, 34 HCs & $31.8(8.0)$ & 59 & - & $\mathrm{URs}=\mathrm{HCs}$ \\
\hline Kulkarni et al. (2010) & $\begin{array}{l}\text { Rey’s Complex Figure } \\
\text { Test }\end{array}$ & Behaviour & 30 URs, $30 \mathrm{HCs}$ & $28.9(7.1)$ & 20 & Siblings & URs $<$ HCs \\
\hline Maziade et al. (2009) & Rey complex figure & Behaviour & 23 URs, 45 HCs & $17.5(4.5)$ & 39 & Offspring & $\mathrm{URs}<\mathrm{HCs}$ \\
\hline Nehra et al. (2014) & $\begin{array}{l}\text { Brief visuospatial } \\
\text { memory test-revised }\end{array}$ & Behaviour & 20 URs, 20 HCs & $36.9(8.6)$ & 15 & Siblings & $\mathrm{URs}=\mathrm{HCs}$ \\
\hline Sobczak et al. (2002) & Picture learning task & Behaviour & 30 URs, 15 HCs & $41.4(2.6)$ & 73 & Mixed & $\begin{array}{l}\text { URs = HCs. FH I vs. FH } \\
\text { II:URs = HCs. Group x Trp } \\
\text { depletion effects: } \\
\text { URs = HCs. }\end{array}$ \\
\hline Tseng et al. (2015) & $\begin{array}{l}\text { Emotional face } \\
\text { encoding }\end{array}$ & Behavioural, fMRI & 13 URs, 37 HCs & $13.7(2.3)$ & 39 & Offspring, sibling & $\begin{array}{l}\downarrow \text { activation in middle } \\
\text { frontal gyrus and } \uparrow \\
\text { activation in } \\
\text { parahippocampal gyrus } \\
\text { during successful vs. } \\
\text { unsuccessful encoding. }\end{array}$ \\
\hline \multicolumn{8}{|c|}{ Reasoning and problem solving } \\
\hline Arslan et al. (2014) & WCST & Behaviour & 55 URs, $32 \mathrm{HCs}$ & $37.7(3.6)$ & 56 & Mixed & $\mathrm{URs}<\mathrm{HCs}$ \\
\hline Bora et al. (2008) & & Behaviour & 34 URs, 25 HCs & $45.7(12.2)$ & 59 & Siblings, parents & URs $<\mathrm{HCs}$ \\
\hline Deveci et al. (2013) & WCST & Behaviour & 30 URs, 37 HCs & $12.3(2.8)$ & 50 & Offspring & $\mathrm{URs}=\mathrm{HCs}$ \\
\hline Doyle et al. (2009) & WCST & Behaviour & 118 URs, 79 HCs & $12.8(4.0)$ & 47 & Siblings & $\mathrm{URs}<\mathrm{HCs}$ \\
\hline Erol et al. (2014) & WCST & Behaviour & 50 URs, $50 \mathrm{HCs}$ & $56.2(6.7)$ & 50 & Parent & $\mathrm{URs}=\mathrm{HCs}$ \\
\hline Frangou et al. (2005) & WCST & Behaviour & 15 URs, 43 HCs & $27.2(8.9)$ & 67 & Offspring & URs $>\mathrm{HCs}$ \\
\hline Frantom et al. (2008) & $\begin{array}{l}\text { WCST, Wechsler Block } \\
\text { Design }\end{array}$ & Behaviour & 19 URs, 19 HCs & $38.3(15.7)$ & 79 & Mixed & $\begin{array}{l}\text { Wechsler Block Design: } \\
\text { URs < HCs, WCST: } \\
\text { URs = HCs }\end{array}$ \\
\hline Juselius et al. (2009) & WCST & Behaviour & 19 URs, 114 HCs & $45.8(1.7)$ & 32 & Co-twins & $\mathrm{URs}=\mathrm{HCs}$ \\
\hline Kim et al. (2015) & WCST & Behaviour & 29 URs, 34 HCs & $31.8(8.0)$ & 59 & - & $\mathrm{URs}=\mathrm{HCs}$ \\
\hline Kosger et al. (2015) & WCST & Behaviour & $\begin{array}{l}24 \mathrm{FD} \mathrm{(} \geq \text { two relatives } \\
\text { with } \mathrm{BD}), 26 \mathrm{SP}(\text { One } \\
\text { first-degree relative), } \\
26 \mathrm{HCs}\end{array}$ & $\begin{array}{l}\text { FP: } 57.5 \text { (7.6); SP: } 56.1 \\
(5.8)\end{array}$ & FP: 46; SP: 54 & Mixed & $\mathrm{FD}<\mathrm{HCs} . \mathrm{SP}<\mathrm{HCs}$ \\
\hline Kulkarni et al. (2010) & WCST, TOL & Behaviour & 30 URs, 30 HCs & $28.9(7.1)$ & 20 & Siblings & $\begin{array}{l}\text { TOL: URs < HCs; WCST: } \\
\text { URs }=\mathrm{HCs}\end{array}$ \\
\hline Li et al. (2015) & WCST & Behaviour & 20 URs, 20 HCs & $40.6(10.5)$ & 45 & Siblings & $\mathrm{URs}=\mathrm{HCs}$ \\
\hline Linke et al. (2013) & $\begin{array}{l}\text { The Intra-Extra } \\
\text { Dimensional Set Shift } \\
\text { Task }\end{array}$ & Behaviour & 22 URs, 22 HCs & $28.0(11.0)$ & 50 & Siblings, offspring & URs $<$ HCs \\
\hline Maziade et al. (2009) & WCST, TOL & Behaviour & 23 URs, $45 \mathrm{HCs}$ & $17.5(4.5)$ & 39 & Offspring & WCST: URs < HCs \\
\hline Nehra et al. (2014) & WCST & Behaviour & 20 URs, $20 \mathrm{HCs}$ & $36.9(8.6)$ & 15 & Siblings & URs $=\mathrm{HCs}$ \\
\hline Sobczak et al. (2002) & Compu-TOL & Behaviour & 30 URs, 15 HCs & $41.4(2.6)$ & 73 & Mixed & $\begin{array}{l}\text { URs }<\text { HCs, FH I vs. FH II: FH } \\
\text { I }<\text { FH II. Group by Trp } \\
\text { depletion: URs }<\text { HCs. }\end{array}$ \\
\hline Sobczak et al. (2003) & Compu-TOL & Behaviour & 30 URs, 15 HCs & $41.0(2.9)$ & 67 & Mixed & $\begin{array}{l}\text { URs = HCs; FH I vs. FH II: FH } \\
\text { I = FH II. Group by Trp } \\
\text { loading: URs = HCs }\end{array}$ \\
\hline Szoke et al. (2006b) & WCST & Behaviour & 51 URs, $50 \mathrm{HCs}$ & $40.4(13.3)$ & 63 & - & URs $=$ HCs \\
\hline
\end{tabular}


Table 1 (Continued)

\begin{tabular}{|c|c|c|c|c|c|c|c|}
\hline Author & Paradigm & Measure & $N$ & Age & Gender (\% female) & UR family status & Finding \\
\hline Szoke et al. (2006a) & WCST & Behaviour & 63 URs, 48 HCs & $40.7(13.0)$ & 61 & - & $\mathrm{URs}=\mathrm{HCs}$ \\
\hline Trivedi et al. (2008) & WCST & Behaviour & 10 URs, $10 \mathrm{HCs}$ & $30.1(11.2)$ & 10 & Siblings & URs $<\mathrm{HCs}$ \\
\hline \multicolumn{8}{|l|}{ Working memory } \\
\hline Antila et al. (2007) & $\begin{array}{l}\text { Digit Span Forward and } \\
\text { Backward (WMS-R), } \\
\text { Visual Span Forward } \\
\text { and Backward } \\
\text { (WMS-R) }\end{array}$ & Behaviour & 40 URs, 55 HCs & $51.4(9.1)$ & 53 & Mixed & $\begin{array}{l}\text { Visual Span Backward task: } \\
\text { URs }<\text { HCs }\end{array}$ \\
\hline Bora et al. (2008) & $\begin{array}{l}\text { Auditory Consonant } \\
\text { Trigrams (ACTT), Digit } \\
\text { Span forward and } \\
\text { Backward, } \\
\text { Letter-Number } \\
\text { Sequencing, }\end{array}$ & Behaviour & 34 URs, 25 HCs & $45.7(12.2)$ & 59 & Siblings, parents & $\begin{array}{l}\text { ACTT total, Digits } \\
\text { backward, Letter-Number } \\
\text { Sequencing: URs }<\text { HCs. } \\
\text { Digits forward: URs = HCs }\end{array}$ \\
\hline Deveci et al. (2013) & $\begin{array}{l}\text { Digit span Forward and } \\
\text { backward (DST) } \\
\text { Auditory consonant } \\
\text { trigram test (ACTT) }\end{array}$ & Behaviour & 30 URs, 37 HCs & $12.3(2.8)$ & 50 & Offspring & $\begin{array}{l}\text { ACTT, digit span forward: } \\
\text { URs }<\text { HCs. Digit span } \\
\text { backward: URs }=\text { HCs }\end{array}$ \\
\hline Doyle et al. (2009) & $\begin{array}{l}\text { WAIS-III: Arithmetic } \\
\text { subset }\end{array}$ & Behaviour & 118 URs, 79 HCs & $12.8(4.0)$ & 47 & Siblings & URs $=\mathrm{HCs}$ \\
\hline Dima et al. (2016) & N-back WM paradigm & Behaviour, fMRI & 25 URs, 46 HCs & $39.7(13.7)$ & 48 & Siblings & $\begin{array}{l}\text { Behavioural: URs }>\text { HCs } \\
\text { (accuracy 3-back } \\
\text { condition); fMRI: } \uparrow \\
\text { activation of bilateral } \\
\text { middle, inferior frontal } \\
\text { gyrus, right temporal gyrus } \\
\text { and bilateral ACC; no } \\
\text { difference in endogenous } \\
\text { connections. }\end{array}$ \\
\hline Frantom et al. (2008) & Digit Span, Spatial Span & Behaviour & 19 URs, $19 \mathrm{HCs}$ & $38.3(15.7)$ & 79 & Mixed & $\mathrm{URs}=\mathrm{HCs}$ \\
\hline Keri et al. (2001) & Span tasks & Behaviour & 20 URs, 20 HCs & $35.1(9.5)$ & 60 & Siblings & $\mathrm{URs}=\mathrm{HCs}$ \\
\hline Kim et al. (2015) & $\begin{array}{l}\text { Digit Span forward and } \\
\text { backward }\end{array}$ & Behaviour & 29 URs, 34 HCs & $31.8(8.0)$ & 59 & - & $\begin{array}{l}\text { Backward digit span: } \\
\text { URS < HCs. Forward Digit } \\
\text { Span: URs = HCs }\end{array}$ \\
\hline Linke et al. (2013) & $\begin{array}{l}\text { WAIS: digit span } \\
\text { subtest }\end{array}$ & Behaviour & 22 URs, 22 HCs & $28.0(11.0)$ & 50 & Siblings, offspring & $\mathrm{URs}=\mathrm{HCs}$ \\
\hline Maziade et al. (2009) & $\begin{array}{l}\text { WAIS Digit span } \\
\text { subtest, Corsi (working } \\
\text { memory) }\end{array}$ & Behaviour & 23 URs, 45 HCs & $17.5(4.5)$ & 39 & Offspring & $\mathrm{URs}=\mathrm{HCs}$ \\
\hline Pattanayak et al. (2012) & $\begin{array}{l}\text { Verbal Working } \\
\text { Memory N-Back Test }\end{array}$ & Behaviour & 20 URs, 20 HCs & $30.7(11.4)$ & 40 & Mixed & $\mathrm{URs}=\mathrm{HCs}$ \\
\hline Thermenos et al. (2011) & $\begin{array}{l}\text { 2-back and 0-back } \\
\text { control WM task }\end{array}$ & Behaviour, fMRI & 10 URs, $10 \mathrm{HCs}$ & $18.4(4.2)$ & 50 & - & $\begin{array}{l}\text { Behaviour: URs }=\text { HCs. } \\
\text { fMRI: } \downarrow \text { activation in } \\
\text { Cerebellar vermis on } 2 \\
\text { back } \uparrow \text { during } 0 \text {-back } \\
\text { condition. } \downarrow \text { activity in } \\
\text { frontopolar region and } \\
\uparrow \text { activity in brain stem, CV, } \\
\text { insula, and amygdala } \\
\text { ( } 0 \text {-back }>\text { fixation contrast). } \\
\text { No differences in OFC. }\end{array}$ \\
\hline
\end{tabular}


Verbal fluency

Christodoulou et al. (2012b)

Costafreda et al. (2009)

Deveci et al. (2013)

Erol et al. (2014)

Frantom et al. (2008)

Juselius et al. (2009)

Keri et al. (2001)

(uzit

Sobczak et al. (2002)

Sobczak et al. (2003)

Zalla et al. (2004)

Executive function

Antila et al. (2007)

Arslan et al. (2014)
Besnier et al. (2009)

Bora et al. (2008)

Christodoulou et al. (2012b)

Deveci et al. (2013)

Deveney et al. (2012)
Controlled oral work association test

Letter fluency Task

Controlled word

association test

Controlled oral work

association

Letter fluency Test,

Semantic fluency Test

Letter fluency

Verbal fluency Test

Verbal fluency task

Verbal fluency test

Verbal fluency test

Verbal fluency

TMT B

SCWT, TMT-B

SCWT

SCWT, TMT B

Hayling sentence

completion task

SCWT

Stop signal task (SST)
Behaviour

fMRI

Behaviour

Behaviour

Behaviour

Behaviour

Behaviour

Behaviour

Behaviour

Behaviour

Behaviour

Behaviour

Behaviour

Behaviour

Behaviour

Behaviour

Behaviour

Behaviour

Behaviour, fMR
17 URs, 23 HCs

7 URs, 48 HCs

30 URs, 37 HCs

50 URs, 50 HCs

19 URs, 19 HCs

19 URs, 114 HCs

20 URs, 20 HCs

30 URs 15 HCs

33 URs, 20 HCs

40 URs, 55 HCs

55 URs, 32 HCs

30 URs, 60 HCs

34 URs, 25 HCs

17 URs, 23 HCs

30 URs, 37 HCs

13 URs, $21 \mathrm{HC}$
38.7 (13.4)

$39.4(15.8)$

$12.3(2.8)$

$56.2(6.7)$

$38.3(15.7)$

$45.8(1.7)$

35.1 (9.5)

$17.5(4.5)$

$41.4(2.6)$

41.0 (2.9)

$37.3(11.0)$

$51.4(9.1)$

$37.7(3.6)$

$41.8(13.8)$

$45.7(12.2)$

$38.7(13.4)$

$12.3(2.8)$

$13.5(1.8)$

76
86
50
50
79
32
60
59
39
73

61

53

56
57

59
Doyle et al. (2009)

Frol et al. (2014)

Frangou (2012)

\section{SCWT}

SCWT, TMT B

Hayling sentence

completion task

SCWT
Behaviour

Behaviour

Behaviour, fMR
118 URs, 79 HC

50 URs, $50 \mathrm{HCs}$

15 URs, 43 HCs

48 URs, 71 HCs
$12.8(4.0)$

$56.2(6.7)$

$27.2(8.9)$

36.5 (13.8)
Mixed

Co-twins

Offspring

Parents

Co-twins

Siblings

offspring

Mixed

Mixed

Parents, siblings

Mixed

Mixed

Parents, siblings

Siblings, parents

Mixed

Offspring

Offspring, siblings

Siblings

Parents
Offspring

Siblings, offspring
URs $<$ HCS

$\mathrm{URs}=\mathrm{HCs}$

URs $<$ HCs

URs $=\mathrm{HCs}$

URs $=\mathrm{HCS}$

$\mathrm{URs}=\mathrm{HCs}$

URs $=$ HCs

URs $=$ HCS

URs $<$ HCs

政;

I = FH II: Group x TRP

depletion: URs $=$ HCs

URs = HCs; FH I vs. FH II: FH

I = FH II: Group by TRP

loading: URs $=\mathrm{HCs}$

$\mathrm{URs}=\mathrm{HCs}$

URs $<$ HCs (\%correct)

URs $=$ HCS

$\mathrm{URs}=\mathrm{HCs}$

URs $<$ HCs

$\mathrm{URs}=\mathrm{HCs}$

URs $=$ HCS

Stop incorrect vs. Stop correct: $\uparrow$ activity in left

putamen. Stop incorrect vs.

Go: $\uparrow$ activity in bilateral

po: $p$ activity in bilateral

putanen. Stop correct vs.

go confrast: no group diff.

co cums and in whole

brain analysis.

URs $<$ HCs

URs $<$ HCs

URs $<$ HCs

Behaviour: URs $=$ HCs.

fMRI: $\downarrow$ engagement of th

superior and inferior

parietal GM, no difference

in caudate and VLPFC 
Table 1 (Continued)

\begin{tabular}{|c|c|c|c|c|c|c|c|}
\hline Author & Paradigm & Measure & $N$ & Age & Gender (\% female) & UR family status & Finding \\
\hline Frantom et al. (2008) & SCWT, TMT B & Behaviour & 19 URs, 19 HCs & $38.3(15.7)$ & 79 & Mixed & $\mathrm{URs}=\mathrm{HCs}$ \\
\hline Hidiroglu et al. (2013) & Stop-Signal Task, SCWT & Behaviour & 30 URs, 33 HCs & $21.0(8.9)$ & 53 & Mixed & $\mathrm{URs}<\mathrm{HCs}$ \\
\hline Juselius et al. (2009) & SCWT, TMT B-A & Behaviour & 19 URs, 114 HCs & $45.8(1.7)$ & 32 & Co-twins & $\begin{array}{l}\text { SCWT: URs < HCs, TMT B-A: } \\
\text { Urs>HCs }\end{array}$ \\
\hline Kim et al. (2012) & The change task & Behaviour, fMRI & 13 URs, $21 \mathrm{HCs}$ & $13.9(2.0)$ & 54 & Siblings, offspring & $\begin{array}{l}\text { Behaviour: no differences. } \\
\text { fMRI: Successful change vs. } \\
\text { Go: } \uparrow \text { activation in right } \\
\text { VLPFC, right inferior } \\
\text { parietal lobe, and left } \\
\text { cerebellar regions; } \\
\text { Unsuccessful change vs. } \\
\text { Go: } \uparrow \text { activation in right } \\
\text { caudate and left } \\
\text { cerebellum; Successful } \\
\text { change vs. Unsuccessful } \\
\text { change: } \uparrow \text { activation in } \\
\text { right VLPFC. }\end{array}$ \\
\hline Kosger et al. (2015) & SCWT, TMT B & Behaviour & $\begin{array}{l}24 \mathrm{FD} \text { ( } \geq \text { two relatives } \\
\text { with } \mathrm{BD}), 26 \mathrm{SP} \text { (One } \\
\text { first-degree relative), } \\
26 \mathrm{HCs}\end{array}$ & $\begin{array}{l}\text { FP: } 57.5 \text { (7.6); SP: } 56.1 \\
(5.8)\end{array}$ & FP: 46; SP: 54 & Mixed & $\begin{array}{l}\text { SCWT: FDs < HCs; } \\
\text { SPs < HCs, TMT B: URs < HCs }\end{array}$ \\
\hline Kulkarni et al. (2010) & SCWT, TMT B & Behaviour & 30 URs, 30 HCs & $28.9(7.1)$ & 20 & Siblings & $\mathrm{URs}=\mathrm{HCs}$ \\
\hline Maziade et al. (2009) & SCWT & Behaviour & 23 URs, 45 HCs & $17.5(4.5)$ & 39 & Offspring & URs $=\mathrm{HCs}$ \\
\hline Pattanayak et al. (2012) & SCWT, TMT B & Behaviour & 20 URs, 20 HCs & $30.7(11.4)$ & 40 & Mixed & $\begin{array}{l}\text { SCWT: URs = HCs, TMT B: } \\
\text { URs < HCs }\end{array}$ \\
\hline Sobczak et al. (2002) & SCWT & Behaviour & 30 URs, 15 HCs & $41.4(2.6)$ & 73 & Mixed & $\begin{array}{l}\text { URs = HCs; FH I vs. FH II: FH } \\
\text { I= FH II; Group x Trp } \\
\text { depletion:.URs = HCs }\end{array}$ \\
\hline Sobczak et al. (2003) & Go/No Go task (GONT) & Behaviour & 30 URs, 15 HCs & $41.0(2.9)$ & 67 & Mixed & $\begin{array}{l}\text { URs = HCS; FH I and FH II: } \\
\text { FH I = FHII;Group x Trp } \\
\text { loading: URs }<\text { HCs }\end{array}$ \\
\hline Szoke et al. (2006b) & TMT B & Behaviour & 51 URs, 50 HCs & $40.4(13.3)$ & 63 & - & $\mathrm{Urs}=\mathrm{HCs}$ \\
\hline Szoke et al. (2006a) b & TMT B & Behaviour & 63 URs, 48 HCs & $40.7(13.0)$ & 61 & - & URs $<\mathrm{HCs}$ \\
\hline Wessa et al. (2015) & Stop signal task (SST) & Behaviour & 27 URs, 29 HCs & $31.8(14.4)$ & 52 & - & $\mathrm{URs}=\mathrm{HCs}$ \\
\hline Zalla et al. (2004) b́ & SCWT & Behaviour & 33 URs, 20 HCs & $37.3(11.0)$ & 61 & Parents, siblings & URs $<$ HCs \\
\hline
\end{tabular}

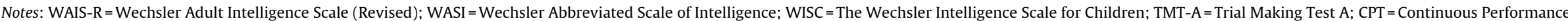

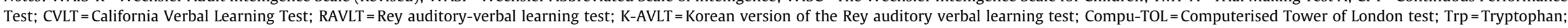

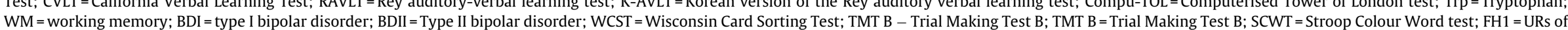
type I BD patients; $\mathrm{FH} 2=$ URs of type II BD patients; URs = unaffected relatives of subjects with $\mathrm{BD} \cdot \mathrm{HCs}=$ Healthy Controls.

a Overlapping sample.

b Partially overlapping sample. 


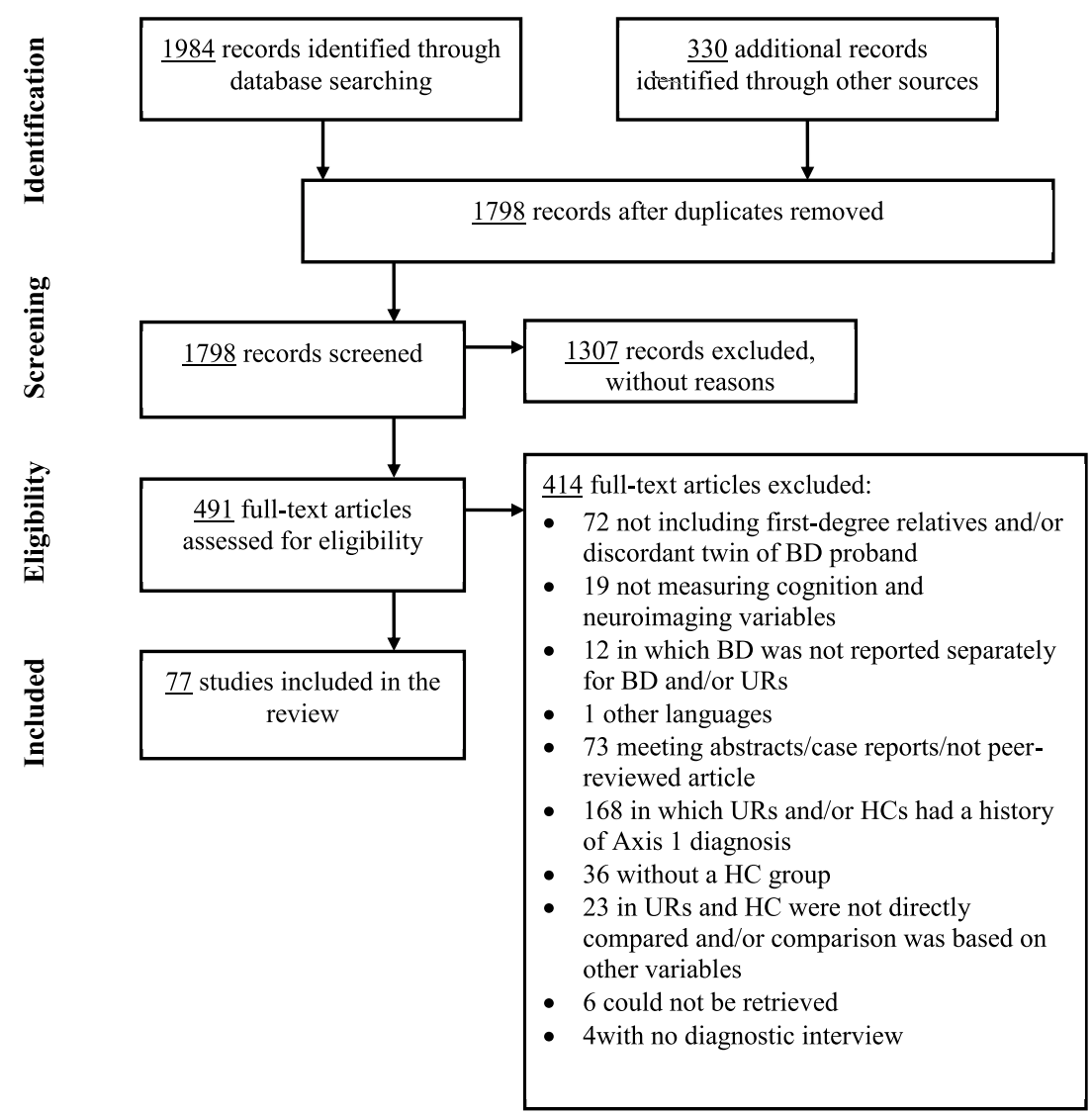

Fig. 1. PRISMA flowchart for study selection for systematic review.

Abbreviations: $\mathrm{BD}$ = bipolar disorder; $\mathrm{HC}=$ healthy controls; $\mathrm{UR}=$ unaffected first-degree relatives of patients with biopalar disorder

2009; Doyle et al., 2009; Patino et al., 2013; Sepede et al., 2012; Sobczak et al., 2003; Trivedi et al., 2008). This discrepancy is likely due to differences between the implemented tests with ceiling effects in some (less demanding) tests (variations of the CPT: e.g., Conners' vs. TOVA, as well as auditory vs. visual measures; see Table 1). Notably, impairment in sustained attention was more often reported in unaffected children than adults at genetic risk (Adleman et al., 2014; Brotman et al., 2009; Deveci et al., 2013; Doyle et al., 2009; Patino et al., 2013). This could potentially indicate acquisition of compensatory strategies with increasing age as a result of CNS maturation. Neuroimaging examination of URs revealed that sustained attention deficits were accompanied by abnormally increased insula and middle cingulate cortex activity (Sepede et al., 2012). The study also showed increased insula and parietal activation in conditions with greater attentional load in URs, suggesting a need for allocation of greater neural resources to sustain attention (i.e., inefficient processing). Taken together, the findings point to deficits in URs on more challenging sustained attention tests, while other tests with lower attentional demands show only neuronal activity differences.

\subsubsection{Verbal learning and memory}

Eleven of the 18 studies (61\%) of verbal memory in URs detected impairments in immediate and delayed recall and in recognition (see Table 1). The discrepancy across studies may be due to differences in BD subtype (I versus II) in URs' affected family member, as poorer verbal memory outcomes have been reported in URs of BD-I relative to BD-II patients (Kosger et al., 2015; Sobczak et al., 2003; Sobczak et al., 2002). Deficits in verbal learning and memory may thus be associated selectively with genetic liability to BD-I, rather than being a general endophenotype of BD.

\subsubsection{Visual learning and memory}

Three studies of visuospatial memory identified impaired performance in URs (Frantom et al., 2008; Kulkarni et al., 2010; Maziade et al., 2009), while an equivalent number of studies $(\mathrm{k}=4)$ with larger sample sizes showed no deficits (Doyle et al., 2009; Kim et al., 2015; Nehra et al., 2014; Sobczak et al., 2002). Regarding visual memory for faces, URs displayed impairments on one (Wechsler Memory Scale-III [WMS-III] Faces I) but not on two other tests (WMS-III Faces II or the Benton Facial Recognition Test) (Frantom et al., 2008). Neuroimaging studies in URs showed hyperactivation in parahippocampal gyrus and hypo-activation in medial PFC regions during encoding of emotional faces (Tseng et al., 2015) as well as hypo-activity in the hippocampus and anterior cingulate cortex (ACC) during memory retrieval of faces (Erk et al., 2014). In summary, there is no convincing evidence for visual learning and memory impairments in URs, although emerging evidence points to aberrant neural activity during encoding and retrieval of faces.

\subsubsection{Reasoning and problem-solving}

Half of studies $(\mathrm{k}=10)$ probing reasoning and problem-solving found worse performance in URs with small to medium effects sizes (see Table 1). One third found impaired performance on the Wisconsin Card Sorting Task (WCST) (Arslan et al., 2014; Bora et al., 2008; Doyle et al., 2009; Kosger et al., 2015; Maziade et al., 2009; Trivedi et al., 2008), while the vast majority of studies using the Tower of London task revealed deficits in URs. Problems with strategic planning may thus be a stronger endophenotype than difficulties with cognitive flexibility (Kulkarni et al., 2010; Maziade et al., 2009; Sobczak et al., 2002). Nevertheless, given the lack of evidence deficits in half of the extant studies suggests that rea- 
Table 2

Eligible studies $(\mathrm{k}=21)$ in which domains related to "hot" cognition were investigated in unaffected relatives and healthy controls.

\begin{tabular}{|c|c|c|c|c|c|c|c|}
\hline Authors & Paradigm & Measure & $N$ & Age & $\begin{array}{l}\text { Gender }(\% \\
\text { female) }\end{array}$ & UR family status & Finding \\
\hline Besnier et al. (2009) & $\begin{array}{l}\text { The Emotional Stroop } \\
\text { Paradigm }\end{array}$ & Behaviour & $\begin{array}{l}30 \text { URs, } 60 \\
\text { HCs }\end{array}$ & $41.8(13.8)$ & 57 & Parents, siblings & $\begin{array}{l}\uparrow \text { emotional interference to disease-associated } \\
\text { words }\end{array}$ \\
\hline Brand et al. (2012) & Affective Go/No-Go & Behaviour & $\begin{array}{l}20 \text { URs, } 20 \\
\text { HCs }\end{array}$ & $39.2(10.5)$ & 70 & Siblings & $\uparrow$ biased responses to negative stimuli \\
\hline Brotman et al. (2008a) & $\begin{array}{l}\text { The Facial Expression } \\
\text { subtest of the Diagnostic } \\
\text { Analysis of Nonverbal } \\
\text { Accuracy Scale }\end{array}$ & Behaviour & $\begin{array}{l}24 \text { URs, } 78 \\
\text { HCs }\end{array}$ & $11.5(4.0)$ & 29 & Offspring, siblings & $\begin{array}{l}\uparrow \text { Misidentification of emotions on both child } \\
\text { and adult faces. }\end{array}$ \\
\hline Brotman et al. (2008b) & $\begin{array}{l}\text { Emotional Expression } \\
\text { Multimorph Task }\end{array}$ & Behaviour & $\begin{array}{l}25 \text { URs, } 36 \\
\text { HC }\end{array}$ & $\begin{array}{l}12.15 \\
(3.05)\end{array}$ & 28 & Offspring, siblings & $\begin{array}{l}\text { URs demanded higher facial expression } \\
\text { intensity to all facial expressions compared to } \\
\text { HCs. }\end{array}$ \\
\hline Brotman et al. (2014) & Parametric faces paradigm & Behaviour, fMRI & $\begin{array}{l}15 \text { URs, } 29 \\
\text { HCs }\end{array}$ & $14.5(2.2)$ & 40 & Offspring, siblings & $\begin{array}{l}\text { Behavioural: angry and happy faces rated less } \\
\text { hostile by URs than HCs. fMRI: } \downarrow \text { amygdala } \\
\text { modulation with increasingly angry faces. No } \\
\text { difference in happy faces. } \downarrow \text { Modulation of } \\
\text { interior frontal gyrus during explicit hostility } \\
\text { ratings, } \uparrow \text { modulation during implicit hostility } \\
\text { ratings. }\end{array}$ \\
\hline Dima et al. (2016) & $\begin{array}{l}\text { Facial affect-recognition } \\
\text { paradigm }\end{array}$ & Behaviour, fMRI & $\begin{array}{l}25 \text { URs, } 46 \\
\text { HCs }\end{array}$ & $39.7(13.7)$ & 48 & Siblings & $\begin{array}{l}\text { Behavioural: URs = HCs; fMRI: } \uparrow \text { connectivity } \\
\text { between the amgydala and the VLPFC, and } \\
\text { between IOG and FG. }\end{array}$ \\
\hline Giakoumaki et al. (2010) & $\begin{array}{l}\text { Startle response to IAPS } \\
\text { images }\end{array}$ & EMG & $\begin{array}{l}19 \text { URs, } 42 \\
\text { HCs }\end{array}$ & $31.8(7.4)$ & - & Siblings & $\begin{array}{l}\text { URs scored pleasant and neutral pictures } \uparrow \text { pos, } \\
\text { and unpleasant pictures } \uparrow \text { neg than HCs. } \\
\text { Relatives has } \downarrow \text { baseline startle amplitude and } \\
\text { blunted attentional and affective startle } \\
\text { modulation compared to HCs. }\end{array}$ \\
\hline Green et al. (2011) & CERQ & Self-report & $\begin{array}{l}124 \text { URs, } 63 \\
\text { HCs }\end{array}$ & $52.3(15.7)$ & 62 & Parents, siblings & $\uparrow$ Habitual rumination and self-blame. \\
\hline Hidiroglu et al. (2013) & $\begin{array}{l}\text { The Balloon Analogue Risk } \\
\text { Task }\end{array}$ & Behavioural & $\begin{array}{l}25 \text { URs, } 30 \\
\text { HCs }\end{array}$ & $40.2(13.4)$ & 68 & Mixed & $\begin{array}{l}\uparrow \text { risk taking propensity (lower adjustment } \\
\text { scores). }\end{array}$ \\
\hline Kanske et al. (2015) & $\begin{array}{l}\text { CERQ, Emotion regulation } \\
\text { to IAPS pictures }\end{array}$ & $\begin{array}{l}\text { Self-report, } \\
\text { Behaviour, fMRI }\end{array}$ & $\begin{array}{l}17 \text { URs, } 17 \\
\text { HCs }\end{array}$ & $36.7(16.3)$ & 47 & Mixed & $\begin{array}{l}\text { Self-report: } \downarrow \text { habitual reappraisal. } \\
\text { Behavioural: UR rated positive images less } \\
\text { positive, and were less successful at } \\
\text { down-regulating positive emotion during } \\
\text { reappraisal. fMRI: } \downarrow \text { down-regulation of } \\
\text { amygdala during reappraisal. Reversed } \\
\text { amygdala-OFC connectivity. }\end{array}$ \\
\hline Kulkarni et al. (2010) & Iowa Gambling Task & Behaviour & $\begin{array}{l}30 \text { URs, } 30 \\
\text { HCs }\end{array}$ & $28.9(7.1)$ & 20 & Siblings & No difference on IGT. \\
\hline Ladouceur et al. (2013) & $\begin{array}{l}\text { The emotional face N-Back } \\
\text { task }\end{array}$ & Behaviour, fMRI & $\begin{array}{l}16 \text { URs, } 15 \\
\text { HCs }\end{array}$ & $14.2(2.3)$ & 44 & Offspring & $\begin{array}{l}\text { Behavioural: no group differences on accuracy } \\
\text { or RT. fMRI: } \uparrow \text { VLPFC activation to happy face } \\
\text { distracters, and } \downarrow \text { VLPFC modulation of right } \\
\text { amygdala to fearful and happy face distracters. }\end{array}$ \\
\hline
\end{tabular}




\begin{tabular}{|c|c|c|c|c|c|c|c|}
\hline Linke et al. $(2012,2013)^{a}$ & $\begin{array}{l}\text { Reversal Learning Task } \\
\text { (RLT), Cambridge Gambling } \\
\text { Task (CGT) }\end{array}$ & $\begin{array}{l}\text { RLT: Behaviour, } \\
\text { fMRI; CGT: } \\
\text { Behaviour, DTI }\end{array}$ & $\begin{array}{l}22 \text { URs, } 22 \\
\text { HCs }\end{array}$ & $28.0(11.0)$ & 50 & Siblings, offspring & $\begin{array}{l}\text { RLT: Behavioural: No diff in RT. fMRI: } \uparrow \\
\text { activation of mOFC to reward, punishment, } \\
\text { and rule reversal, } \uparrow \text { activation in amygdala to } \\
\text { reward and rule reversal. CGT: } \uparrow \text { risk taking } \\
\text { (i.e., points gambled) in URs. Risk taking } \\
\text { correlated negatively with FA in the anterior } \\
\text { limb of internal capsule for URs and HCs. }\end{array}$ \\
\hline Olsavsky et al. (2012) & $\begin{array}{l}\text { Facial emotion processing } \\
\text { paradigm }\end{array}$ & Behaviour, fMRI & $\begin{array}{l}13 \text { URs, } 56 \\
\text { HCs }\end{array}$ & $14.0(2.4)$ & 46 & Offspring, siblings & $\begin{array}{l}\text { Behavioural: no group diff in afraid ratings of } \\
\text { fearful and happy faces. fMRI: } \uparrow \text { activation in } \\
\text { right amygdala to fearful faces. }\end{array}$ \\
\hline Pavlickova et al. (2014) & $\begin{array}{l}\text { The Domain-Specific } \\
\text { Risk-Taking Test } \\
\text { (DOSPERT) }\end{array}$ & Self-report & $\begin{array}{l}30 \text { URs, } 30 \\
\text { HCs }\end{array}$ & $15.9(1.9)$ & 57 & Offspring & No differences in risk-taking. \\
\hline Seidel et al. (2012) & $\begin{array}{l}\text { Facial emotion recognition } \\
\text { paradigm }\end{array}$ & Behaviour & $\begin{array}{l}21 \text { URs, } 21 \\
\text { HCs }\end{array}$ & $38.4(17.7)$ & 48 & - & $\begin{array}{l}\downarrow \text { accuracy in emotion recognition (lowest } \\
\text { accuracy for sad faces). } \downarrow \text { RT to emotional faces. } \\
\text { (lowest to fearful faces). }\end{array}$ \\
\hline Sepede et al. (2015) & $\begin{array}{l}\text { Emotion processing to } \\
\text { negative IAPS pictures }\end{array}$ & fMRI & $\begin{array}{l}22 \text { URs, } 24 \\
\text { HCs }\end{array}$ & $31.5(7.3)$ & 68 & Offspring, siblings & $\begin{array}{l}\uparrow \text { activation in left insula and right lingual } \\
\text { gyrus, } \downarrow \text { activation in right supramarginal } \\
\text { gyrus, pre-supplementary motor area, and } \\
\text { right superior frontal gyrus to negative images. }\end{array}$ \\
\hline Singh et al. (2014b) & $\begin{array}{l}\text { Monetary Incentive Delay } \\
\text { Task }\end{array}$ & $\begin{array}{l}\text { Voxel-wise } \\
\text { neuroimaging }\end{array}$ & $\begin{array}{l}20 \text { URs, } 25 \\
\text { HCs }\end{array}$ & $12.7(2.9)$ & 65 & Offspring & $\begin{array}{l}\uparrow \text { activation in left lateral OFC to reward, } \downarrow \\
\text { activation in pregenual cingulate to losses, and } \\
\text { weaker functional connectivity between the } \\
\text { pregenual cingulate and the right VLPFC while } \\
\text { anticipating rewards. }\end{array}$ \\
\hline Surguladze et al. (2010) & $\begin{array}{l}\text { Facial emotion processing } \\
\text { paradigm }\end{array}$ & fMRI & $\begin{array}{l}20 \text { URs, } 20 \\
\text { HCs }\end{array}$ & $43.0(13.8)$ & 40 & - & $\begin{array}{l}\uparrow \text { activation in mPFC to fearful and happy faces. } \\
\uparrow \text { activity in left amygdala to intensely happy } \\
\text { faces. }\end{array}$ \\
\hline Wessa et al. (2015) & Cambridge Gambling Task & Behaviour & $\begin{array}{l}27 \text { URs, } 29 \\
\text { HCs }\end{array}$ & $31.8(14.4)$ & 52 & - & $\uparrow$ delay aversion \\
\hline
\end{tabular}

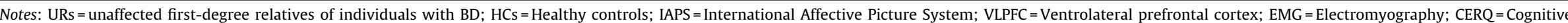

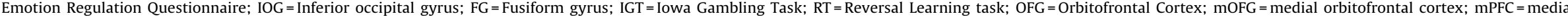
prefrontal cortex.

a Overlapping sample. 
soning and problem solving difficulty is not a strong candidate endophenotype for BD.

\subsubsection{Working memory}

Of the 12 studies of working memory (WM), only four found impairments in URs (Antila et al., 2007; Bora et al., 2008; Deveci et al., 2013; Kim et al., 2015). Of these studies, the majority found impairments on the digit/visual span backward test, suggesting that WM tests with greater cognitive demands are needed to uncover WM deficits in URs (i.e., avoiding ceiling effects). Notably, most of these studies specifically investigated URs of BD-I patients, and the findings could thus reflect a specific association between the genetic liability to the BD I subtype. One neuroimaging study revealed reduced activity in the (task-relevant) frontopolar cortex coupled with increased activity in (task-irrelevant) limbic regions and insula during $\mathrm{N}$-back WM performance (Thermenos et al., 2011). However, another study using a similar N-back task found no differences between URs and HCs on WM network activity or connectivity (Dima et al., 2016). Overall, these findings point to no conclusive association between WM impairment and genetic liability to BD.

\subsubsection{Verbal fluency}

Three of 12 studies found impairments on verbal fluency in URs with large effect sizes (ranging from 0.6-1.0). The observed impairments were specific to phonemic fluency (i.e., the generation of words starting with a specific letter) as opposed to semantic category (Christodoulou et al., 2012a; Deveci et al., 2013; Maziade et al., 2009). Nevertheless, most studies $(k=9)$ found no verbal fluency decrease in URs (Erol et al., 2014; Frantom et al., 2008; Juselius et al., 2009; Keri et al., 2001; Kim et al., 2015; Sobczak et al., 2003; Sobczak et al., 2002; Zalla et al., 2004). Consistent with this, a small fMRI twin study of seven unaffected co-twins, found no difference in neural activity maps during verbal fluency performance. However, this may be due to type II error given the suboptimal sample size for detection of differences in neural activity between groups (Costafreda et al., 2009). Taken together, the majority of behavioural and neuroimaging studies found no consistent verbal fluency impairment in URs.

\subsubsection{Executive function}

Executive function in URs has been examined extensively in a total of 25 studies. Almost half of studies $(k=12)$ found executive dysfunction in URs (using TMT-B and the Stroop Colour Word Task (SCWT), whereas the remaining half $(\mathrm{k}=13)$ found no impairment (see Table 1). No consistent pattern of differences in methods, tests, or samples could account for these discrepancies. Despite consistent findings of aberrant neural activity during performance on executive function tests, the direction and location of these changes were conflicting. Specifically, some studies found increased activity in the striatum, ventrolateral prefrontal cortex (VLPFC), and interior parietal lobe (Deveney et al., 2012; Kim et al., 2012), while others showed reduced superior and inferior parietal activity (with no differences in caudate and VLPFC) (Frangou, 2012). These discrepancies may be due to differences in paradigms used (i.e., the go-no go task and the stop signal task vs. the SCWT) as these tap into disparate aspects of executive function (i.e., response selection vs. response execution). Taken together, behavioural findings have been inconsistent, whereas neuroimaging findings point to aberrant task-related fronto-parietal activity in URs.

\subsubsection{Interim summary}

Within the 'cold' cognition domains, evidence indicates that the most promising neurocognitive endophenotypes for $\mathrm{BD}$ are deficits in verbal memory, problem-solving, and executive function. Despite some degree of variability in behavioural measures of cognition, neuroimaging studies provided relatively consistent evidence for aberrant neural activity in task-relevant neural circuits even when no behavioural differences were identified. Functional neuroimaging measures thus seem to be a more sensitive assay of abnormal brain function than behavioural read-outs.

\section{2. 'Hot' cognition}

Table 2 displays the 'hot' cognition studies performed in UDs of BD patients. A total of 21 studies investigated 'hot' cognition (Besnier et al., 2009; Brand et al., 2012; Brotman et al., 2014; Brotman et al., 2008a; Brotman et al., 2008b; Dima et al., 2016; Giakoumaki et al., 2010; Green et al., 2011; Hidiroglu et al., 2013; Kanske et al., 2015; Kulkarni et al., 2010; Ladouceur et al., 2013; Linke et al., 2013; Linke et al., 2012; Olsavsky et al., 2012; Pavlickova et al., 2014; Seidel et al., 2012; Sepede et al., 2015; Singh et al., 2014b; Surguladze et al., 2010; Wessa et al., 2015) involving 545 UR and $693 \mathrm{HC}$ participants. Of these, 10 examined only behavioural measures, while 11 also incorporated imaging assessments. The studies explored following aspects of 'hot' cognition: emotion processing and regulation, implicit processing of emotional information, as well as risk-taking, reward and punishment processing (for study details see Table 2).

\subsubsection{Emotion processing and regulation}

Five studies showed consistent non-specific deficits in the recognition of facial displays of emotion in URs. In particular, unaffected children displayed deficits in the recognition of happiness, sadness, anger, and fear (Brotman et al., 2008a), and required higher emotion intensity to identify those facial expressions (Brotman et al., 2008b). Similarly, adult URs showed general slowing and reduction in accuracy during identification of emotional facial expressions (Seidel et al., 2012). These facial expression recognition problems in URs have been shown to be accompanied by aberrant frontal and/or limbic activation (Brotman et al., 2014; Dima et al., 2016; Olsavsky et al., 2012; Surguladze et al., 2010). Unaffected youths have been shown to exhibit decreased amygdala and inferior frontal gyrus response to angry facial expressions (Brotman et al., 2014) and exaggerated amygdala response to fearful (but not happy) faces (Dima et al., 2016; Olsavsky et al., 2012). In contrast, a study of adult URs showed exaggerated amygdala response to happy (but not fearful) faces coupled with increased mPFC reactivity to both happy and fearful faces (Surguladze et al., 2010). Taken together, there is consistent evidence for aberrant frontolimbic activity to emotional faces in URs. The discrepancy in the direction the activity changes may be due to different experimental paradigms across studies (i.e., passive viewing vs. task-directed processing of faces), or could indicate age-related differences in individuals at familial risk for BD.

One fMRI study using emotion-laden picture stimuli reveaked a general blunted startle response in URs, which may indicate aberrant affective reactivity to both pleasant and unpleasant emotional pictures (Giakoumaki et al., 2010). Such abnormal response to emotional pictures is consistent with the demonstration of abnormal neural responses, including hyper-activation in insula and hypoactivation in parietal cortex to negative emotional picture stimuli (Sepede et al., 2015).

A handful of studies have investigated emotion regulation in URs using behavioural measures $(\mathrm{k}=1)$ and fMRI $(\mathrm{k}=1)$ or self-report measures $(k=2)$. The studies provide emerging evidence for more 'positive' ratings of positive pictures in URs relative to HCs, and deficient down-regulation of positive emotion during reappraisal of these images (Kanske et al., 2015). They also show increased amygdala activity coupled with reduced functional coupling between cortico-limbic regions during reappraisal of emotional pictures of either valence (Kanske et al., 2015). These findings corroborate with 
the observation of reduced prefrontal top-down control of limbic reactivity to emotional face distracters during a WM paradigm (Ladouceur et al., 2013) and point to deficient cortico-limbic coupling as a marker of genetic risk for BD. On self-report measures of habitual emotion regulation strategies, a large-scale study found abnormally increased engagement in self-blame and rumination in URs (Green et al., 2011), while another study found that URs were less likely to engage in cognitive reappraisal (Kanske et al., 2015).

Taken together, there is emerging evidence for deficits in the recognition of facial expressions and in the ability to dampen emotional response to positively valenced emotional information in URs, which is accompanied by abnormal functional connnectivity between prefrontal and limbic regions. There is also some (albeit very limited) evidence for dysfunctional habitual emotional regulation strategies in URs.

\subsubsection{Cognitive interference of emotional stimuli}

Two studies found that URs are more easily distracted by task-irrelevant emotional stimuli than HCs (Besnier et al., 2009; Brand et al., 2012). Specifically, in the Emotional Stroop task, they responded slower to depressive-related words (e.g., sad, depressed) and committed more errors for mania-related words (e.g., cheerful, agitation) (Besnier et al., 2009), which is indicative of greater emotional distractibility, irrespective of valence. URs also displayed greater obstruction of attentional resources by negatively valenced words in an affective Go-NoGo task (Brand et al., 2012). Taken together, these findings indicate that attentional interference by task-irrelevant emotional stimuli is a promising candidate endophenotype for BD.

\subsubsection{Risk-taking, reward and punishment processing}

Five studies have found increased risk-taking behaviour in URs (Hidiroglu et al., 2013; Linke et al., 2013; Linke et al., 2012; Singh et al., 2014b; Wessa et al., 2015), whereas two studies found no differences (Kulkarni et al., 2010; Pavlickova et al., 2014). These showed lower adjustment scores after losses, suggesting that genetic liability for BD may be linked to a reduced ability to modulate risky activity in the face of certain types of acute stressors (Hidiroglu et al., 2013). At the neural level, white matter integrity in thalamocortical and frontolimbic tracts correlated negatively with risk taking (Linke et al., 2013). Moreover, children at familial risk for BD showed increased orbitofrontal cortex (OFC) activation in response to reward, and decreased pregenual cingulate activation during anticipation of loss on a monetary incentive delay task, which may indicate an increased sensitivity to rewards vs. losses. They also exhibited weaker connectivity between the pregenual cingulate and the VLPFC during reward anticipation, but a stronger connectivity between these regions during anticipation of loss (Singh et al., 2014b). These findings point to deficient top-down regulatory mechanisms during anticipation of reward and excessive emotion regulation during anticipation of losses. Furthermore, URs of BD-I patients show exaggerated activation of the medial OFC in response to reward, punishment, and rule reversal (Linke et al., 2012), and amygdala hyperactivation during rule reversal (Linke et al., 2012). Taken together, these results suggest aberrant frontostriatal activation to reward and losses, which may be a putative neuroimaging endophenotype for BD.

\subsubsection{Interim summary}

URs exhibit abnormalities at the behavioural and neural levels of emotion processing and regulation, including deficits in the recognition of emotional faces, impaired ability to downregulate positive emotion and a greater attentional interference by emotional stimuli. However, the most consistent evidence for abnormalities in 'hot' cognition is derived from fMRI studies that showed aberrant fronto-limbic activation to different types of emo- tional stimuli. During reward processing, URs were found to exhibit weaker functional vlPFC-pregenual cingulate connectivity while anticipating rewards, which could underlie a failure to downregulate positive emotion during reward anticipation, although further replication is necessary.

\subsection{Neuroimaging}

\subsubsection{Structural neuroimaging abnormalities}

As seen from Table 3, 15 studies including 557 URs and 858 HCs (Bauer et al., 2014; Eker et al., 2014; Frangou, 2012; Frazier et al., 2007; Gunde et al., 2011; Hajek et al., 2015; Hajek et al., 2013; Ladouceur et al., 2008; Linke et al., 2013; Matsuo et al., 2012; Sandoval et al., 2016; Saricicek et al., 2015; Teixeira et al., 2014; Tighe et al., 2012; Versace et al., 2010) analysed structural brain abnormalities in URs; nine reported results for grey matter and eight reported results for white matter (for details see Table 3 ).

3.3.1.1. Grey matter. Five of the nine studies found reduced grey matter volume in URS compared to HCs in the OFC (Eker et al., 2014; Sandoval et al., 2016), insula (Matsuo et al., 2012; Sandoval et al., 2016), and cerebellum (Eker et al., 2014; Saricicek et al., 2015), respectively. On the contrary, four studies reported increased grey matter volume in the dorsolateral prefrontal cortex (DLPFC) (Eker et al., 2014), inferior frontal gyrus (Hajek et al., 2013; Saricicek et al., 2015), insula, and cerebellum (Frangou, 2012) in URs compared to HCs. One study reported increased volume of the parahippocampal gyrus extending into hippocampus in unaffected offspring, but no significant differences in amygdala volume (Ladouceur et al., 2008), whereas another other study found increased volume of the amygdala but not hippocampal volumes in unaffected offspring (Bauer et al., 2014). Three studies showed no volume difference in orbitomedial prefrontal cortex (OMPFC), ventromedial prefrontal cortex (VMPFC), or DLPFC (Bauer et al., 2014; Ladouceur et al., 2008; Matsuo et al., 2012). Taken together, the findings regarding grey matter volume were conflicting and inconclusive. We could not identify a consistent pattern of differences in methods or samples that could account for these discrepancies.

3.3.1.2. White matter abnormalities. Of the eight studies, one study found decreased white matter volumes of the right (but not left) medial frontal gyrus in URs (Matsuo et al., 2012). Relatives could be distinguished from HCs in several white matter tracts adjacent to areas involved in the processing of emotions (ventral PFC regions, cingulate gyrus, superior middle temporal gyrus, precuneus, and posterior regions of the occipital lobe) (Hajek et al., 2015). Other studies found bilaterally compromised white matter integrity in the superior longitudinal fasciculus (Frazier et al., 2007), the inferior longitudinal fasciculus, the corpus callosum (Versace et al., 2010), and the internal capsule and unincate fasiculus (Linke et al., 2013). However, two studies found no white matter hyperintensities (WMH) in URs (Gunde et al., 2011; Tighe et al., 2012), despite intermediate volume of WMH relative to HCs and patients with BD (Tighe et al., 2012), and one study showed no white matter abnormalities in any brain regions (Teixeira et al., 2014). This large discrepancy in the results may be due to different diffusion measures (fractional anisotropy, mean, radial or longitudinal-diffusivity; for details see Table 3), as well as the analytic approaches used. Notwithstanding the discrepancies, the findings point to the presence of some local reductions in white matter integrity in URs.

\subsubsection{Resting state functional connectivity}

Four studies examined functional connectivity during resting state in URs (Li et al., 2015; Lui et al., 2015; Meda et al., 2012; Singh et al., 2014a) (for an overview see Table 3). The most consistent 
Table 3

Functional and structural neuroimaging and electrophysiological studies included in systematic review.

\begin{tabular}{|c|c|c|c|c|c|c|c|}
\hline Author & Paradigm & Measure & $N$ & Age & $\begin{array}{l}\text { Gender } \\
\text { (\% } \\
\text { female) }\end{array}$ & UR family status & Main Findings \\
\hline Bauer et al. (2014) & $\begin{array}{l}\text { GM volume } \\
\text { (Surface based) }\end{array}$ & sMRI & $\begin{array}{l}18 \text { URs, } 45 \\
\text { HCs }\end{array}$ & $10.5(3.4)$ & 50 & Offspring & $\begin{array}{l}\uparrow \text { GM volume in the right } \\
\text { amygdala. No group differences in } \\
\text { GM volume of striatum, caudate, } \\
\text { hippocampus, cingulate cortex, } \\
\text { temporal regions, fusiform gyrus or } \\
\text { PFC }\end{array}$ \\
\hline Eker et al. (2014) & GM volume (VBM) & SMRI & $\begin{array}{l}28 \text { URs, } 30 \\
\text { HCs }\end{array}$ & $34.9(9.4)$ & 60 & Siblings & $\begin{array}{l}\downarrow \text { GM volume in the left } \\
\text { orbitofrontal region and right } \\
\text { cerebellum, and } \uparrow \text { GM volume in } \\
\text { the left DLPFC }\end{array}$ \\
\hline Frangou (2012) & GM volume (VBM) & SMRI & $\begin{array}{l}48 \text { URs, } 71 \\
\text { HCs }\end{array}$ & $36.5(13.8)$ & 52 & Siblings, offspring & $\uparrow$ volume of vermis and insula. \\
\hline Frazier et al. (2007) & FA & DTI & $\begin{array}{l}7 \text { URs, } 8 \\
\text { HCs }\end{array}$ & $8.9(3.0)$ & 43 & Siblings, offspring & $\begin{array}{l}\downarrow \text { FA bilaterally in the superior } \\
\text { longitudinal fasciculus }\end{array}$ \\
\hline $\begin{array}{l}\text { Giakoumaki et al. } \\
\text { (2007) }\end{array}$ & $\begin{array}{l}\text { Acoustic startle } \\
\text { reactivity, prepulse } \\
\text { inhibition of startle } \\
\text { response }\end{array}$ & EMG & $\begin{array}{l}19 \text { URs, } 17 \\
\text { HCs }\end{array}$ & $31.6(7.5)$ & - & Siblings & $\begin{array}{l}\downarrow \text { prepulse inhibition. No } \\
\text { difference between groups in } \\
\text { startle reactivity and habituation. }\end{array}$ \\
\hline Gunde et al. (2011) & $\begin{array}{l}\text { Number of white } \\
\text { matter } \\
\text { hyperintensities }\end{array}$ & SMRI & $\begin{array}{l}44 \text { URs, } 49 \\
\text { HCs }\end{array}$ & $19.8(3.6)$ & 64 & Offspring & $\begin{array}{l}\text { No difference in number of white } \\
\text { matter hyperintensities }\end{array}$ \\
\hline Hajek et al. (2013)a & GM volume (VBM) & SMRI & $\begin{array}{l}30 \text { URs, } 31 \\
\text { HCs }\end{array}$ & $19.5(3.1)$ & 67 & Offspring & $\begin{array}{l}\uparrow \text { right inferior frontal gyrus } \\
\text { volume. No other group difference } \\
\text { in GM volume. }\end{array}$ \\
\hline Hajek et al. (2015) ${ }^{a}$ & $\begin{array}{l}\text { WM, GM (machine } \\
\text { learning) }\end{array}$ & SMRI & $\begin{array}{l}45 \text { URs, } 45 \\
\text { HCs }\end{array}$ & $20.1(3.6)$ & 64 & Offspring & $\begin{array}{l}\text { URs were distinguished from HCs } \\
\text { in bilateral white matter tracts } \\
\text { adjacent to the inferior/middle } \\
\text { frontal regions, cingulate gyrus, } \\
\text { superior/middle temporal gyrus, } \\
\text { precuneus, and posterior regions in } \\
\text { occipital lobe. No group difference } \\
\text { in SVM and GPC for grey matter. }\end{array}$ \\
\hline $\begin{array}{l}\text { Katsanis et al. } \\
\text { (1996) }\end{array}$ & $\begin{array}{l}\text { Visual event } \\
\text { related potentials } \\
(\text { ERP) }\end{array}$ & EEG & $\begin{array}{l}31 \text { URs, } 113 \\
\text { HCs }\end{array}$ & $37.0(14.6)$ & 48 & - & $\begin{array}{l}\text { No difference in amplitude, } \\
\text { variability and reducing vs. } \\
\text { augmentation of N1, P1 and P2. }\end{array}$ \\
\hline $\begin{array}{l}\text { Ladouceur et al. } \\
\text { (2008) }\end{array}$ & GM volume (VBM) & SMRI & $\begin{array}{l}20 \text { URs, } 22 \\
\text { HCs }\end{array}$ & $13.0(2.7)$ & 55 & Offspring & $\begin{array}{l}\uparrow \text { volume of left parahippocampal } \\
\text { gyri extending into left } \\
\text { hippocampus. No group difference } \\
\text { in amygdala and orbitomedial PFC } \\
\text { volume }\end{array}$ \\
\hline Li et al. (2015) & $\begin{array}{l}\text { Functional } \\
\text { connectivity }\end{array}$ & rfMRI & $\begin{array}{l}20 \text { URs, } 20 \\
\text { HCs }\end{array}$ & $40.6(10.5)$ & 45 & Siblings & $\begin{array}{l}\uparrow \text { connectivity between right } \\
\text { DLPFC-bilateral amygdala }\end{array}$ \\
\hline Linke et al. (2013) & $\begin{array}{l}\text { Fractional } \\
\text { Anisotrophy (FA), } \\
\text { RD and LD }\end{array}$ & DTI & $\begin{array}{l}22 \text { URs, } 22 \\
\text { HCs }\end{array}$ & $28.0(11.0)$ & 50 & Siblings, offspring & $\begin{array}{l}\downarrow F A \text { and } \uparrow R D \text { in the right anterior } \\
\text { limb of the internal capsule and } \downarrow \\
\text { FA in the right uncinate fasciculus. } \\
\text { No group difference in Corpus } \\
\text { Callosum. }\end{array}$ \\
\hline Lui et al. (2015) & $\begin{array}{l}\text { Amplitude of low } \\
\text { frequency } \\
\text { fluctuations (ALFF), } \\
\text { functional } \\
\text { connectivity }\end{array}$ & rfMRI & $\begin{array}{l}28 \text { URs, } 59 \\
\text { HCs }\end{array}$ & $37.0(15.0)$ & 61 & - & $\begin{array}{l}\uparrow \text { Functional connectivity between } \\
\text { the left precentral/postcentral } \\
\text { gyrus and bilateral caudate. No } \\
\text { other difference in ALFF } \\
\text { abnormalities. }\end{array}$ \\
\hline $\begin{array}{l}\text { Matsuo et al. } \\
(2012)\end{array}$ & $\begin{array}{l}\text { WM and GM } \\
\text { volume (VBM) }\end{array}$ & SMRI & $\begin{array}{l}20 \text { URs, } 40 \\
\text { HCs }\end{array}$ & $46.2(10.7)$ & 75 & Mixed & $\begin{array}{l}\downarrow \text { left anterior insular GM volumes } \\
\text { and } \downarrow \text { WM volumes of the right } \\
\text { medial frontal gyrus. No group } \\
\text { differences in total GM, WM or } \\
\text { brain volumes. No group } \\
\text { differences in GM volume of } \\
\text { VMPFC, DLPFC, ACC, striatum, } \\
\text { hippocampus }\end{array}$ \\
\hline Meda et al. (2012) & $\begin{array}{l}\text { Functional network } \\
\text { connectivity }\end{array}$ & rfMRI & $\begin{array}{l}52 \text { URs, } 118 \\
\text { HCs }\end{array}$ & $40.6(13.0)$ & 65 & - & $\begin{array}{l}\downarrow \text { connectivity between the } \\
\text { Fronto/occipital network and the } \\
\text { Anterior default mode/prefrontal } \\
\text { network, and between the } \\
\text { Meso/paralimbic network and the } \\
\text { fronto-temporal/paralimbic } \\
\text { network. No difference between } \\
\text { the meso/paralimbic and the } \\
\text { fronto-temporal/paralimbic } \\
\text { networks. }\end{array}$ \\
\hline
\end{tabular}


Table 3 (Continued)

\begin{tabular}{|c|c|c|c|c|c|c|c|}
\hline Author & Paradigm & Measure & $N$ & Age & $\begin{array}{l}\text { Gender } \\
(\% \\
\text { female) }\end{array}$ & UR family status & Main Findings \\
\hline $\begin{array}{l}\text { Pierson et al. } \\
(2000)\end{array}$ & $\begin{array}{l}\text { Event related } \\
\text { potentials (ERP). } \\
\text { Auditory oddball } \\
\text { task, N100, N200, } \\
\text { P200, P300, targets } \\
\text { and non-targets }\end{array}$ & EEG & $\begin{array}{l}19 \text { URs, } 19 \\
\text { HCs }\end{array}$ & $26.2(6.6)$ & 63 & Siblings, offspring & $\begin{array}{l}\downarrow \text { amplitude and longer latency, } \\
\text { and a lack of hemispheric } \\
\text { dominance of P300, longer } \\
\text { latencies of N100 target and lower } \\
\text { amplitude for N100 non-target. } \\
\text { Behaviourally: } \uparrow \text { RT and RT-N200 } \\
\text { latency in both midline and } \\
\text { hemispheres }\end{array}$ \\
\hline $\begin{array}{l}\text { Sandoval et al. } \\
\text { (2016) }\end{array}$ & GM volume & SMRI & $\begin{array}{l}12 \text { URs, } 18 \\
\text { HCs }\end{array}$ & $43.4(19.4)$ & 58 & Mixed & $\begin{array}{l}\downarrow \text { right and left PFC, right and left } \\
\text { globus pallidum, right and left OFC, } \\
\text { right and left insular cortex, left } \\
\text { amygdala, right middle temporal } \\
\text { gyrus, and total cortex. }\end{array}$ \\
\hline $\begin{array}{l}\text { Saricicek et al. } \\
\text { (2015) }\end{array}$ & GM volume (VBM) & SMRI & $\begin{array}{l}25 \text { URs, } 29 \\
\text { HCs }\end{array}$ & $32.1(11.0)$ & 54 & Mixed & $\begin{array}{l}\uparrow \text { left and right interior frontal gyri, } \\
\text { left parahippocampal gyrus, left } \\
\text { supramarginal gyrus; } \downarrow \\
\text { cerebellum, vermis }\end{array}$ \\
\hline Singh et al. (2014a) & $\begin{array}{l}\text { Functional } \\
\text { connectivity }\end{array}$ & rfMRI & $\begin{array}{l}24 \text { URs, } 25 \\
\text { HCs }\end{array}$ & $12.3(3.0)$ & 67 & Offspring & $\begin{array}{l}\uparrow \text { connectivity within the left } \\
\text { executive control Network, spec. } \\
\text { VLPFC, } \downarrow \text { connectivities between } \\
\text { the left amygdala and pregenual } \\
\text { cingulate, between the subgenual } \\
\text { cingulate and supplementary } \\
\text { motor context, and between the } \\
\text { left VLPFC and left caudate. } \uparrow \\
\text { connectivity between left VLPFC } \\
\text { and left superior parietal lobule. No } \\
\text { difference in right executive } \\
\text { control network, ventral default } \\
\text { mode network and default mode } \\
\text { network. No differences in } \\
\text { connectivity between right } \\
\text { amygdala or right VLPFC. URs had a } \\
\text { significant decrease of FC between } \\
\text { left VLPFC and left caudate with } \\
\text { increasing family chaos. }\end{array}$ \\
\hline $\begin{array}{l}\text { Teixeira et al. } \\
(2014)\end{array}$ & $\begin{array}{l}\text { FA, MD, RD, AD } \\
\text { (TBSS) }\end{array}$ & DTI & $\begin{array}{l}18 \text { URs, } 20 \\
\text { HCs }\end{array}$ & $12.7(3.1)$ & 50 & Offspring & $\begin{array}{l}\text { No difference in } \mathrm{FA}, \mathrm{MD}, \mathrm{RD} \text { or } \mathrm{AD} \\
\text { between groups }\end{array}$ \\
\hline Tighe et al. (2012) & $\begin{array}{l}\text { Volume of white } \\
\text { matter } \\
\text { hyperintensities }\end{array}$ & SMRI & $\begin{array}{l}7 \text { URs, } 32 \\
\text { HCs }\end{array}$ & & & & No difference between groups. \\
\hline $\begin{array}{l}\text { Versace et al. } \\
\text { (2010) }\end{array}$ & $\begin{array}{l}\text { FA, RD (transverse } \\
\text { diffusivity), L1 } \\
\text { (Longitudinal } \\
\text { diffusivity) }\end{array}$ & DTI & $\begin{array}{l}20 \text { URs, } 25 \\
\text { HCs }\end{array}$ & $13.2(2.5)$ & 45 & Offspring & $\begin{array}{l}\text { Main group effect: } \uparrow \text { FA and } \downarrow \text { RD in } \\
\text { Corpus Callosum, } \downarrow \text { RD in right ILF } \\
\text { in the temporal lobe and } \uparrow \text { L1 in } \\
\text { right ILF in the visual cortex. Age x } \\
\text { group interaction: left corpus } \\
\text { callosum showed a decrease of FA } \\
\text { with age in URs compared to an } \\
\text { increase in FA with age in HC. A } \\
\text { nearby region showed an increase } \\
\text { of RD with age in URs compared to } \\
\text { a decrease of RD with age in HC. ILF } \\
\text { in the temporal lobe showed a } \\
\text { decrease of RD with age in HCs but } \\
\text { no relation of age and RD in URs. } \\
\text { Right IFL in the visual cortex } \\
\text { showed a decrease of L1 with age } \\
\text { in high risk compared to an } \\
\text { increase of L1 with age in healthy } \\
\text { controls. }\end{array}$ \\
\hline
\end{tabular}

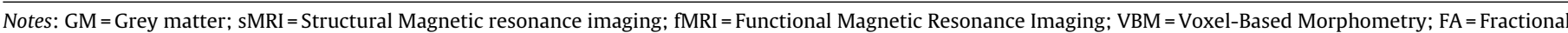

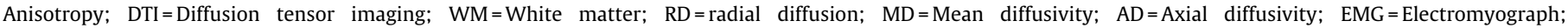

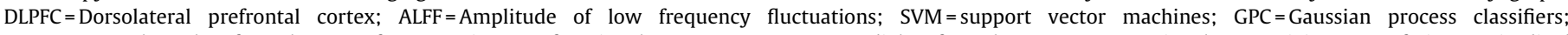

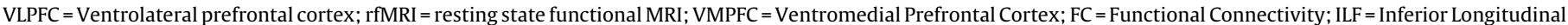
Fasciculus; TBSS = Tract-Based Spatial Statistics.

a Partially overlapping samples.

finding was differences in functional connectivity within regions of the frontostriatal circuitry (Lui et al., 2015; Singh et al., 2014a), although the direction of the abnormalities differed between these studies. In particular, one study showed reduced connectivity between fronto-occipital regions and the anterior default mode- prefrontal network, and between the meso-paralimbic network and fronto-temporal-paralimbic regions (Meda et al., 2012). In contrast, another study found no activity difference within the default mode network (DMN) (Singh et al., 2014a) - a network of medial brain regions that is active during rest and subserves 
thought wandering and internal focus (Cha et al., 2014; Raichle and Snyder, 2007). Finally, one study found increased connectivity between dIPFC and amygdala bilaterally in URs during resting state (Li et al., 2015). Discrepancies regarding the direction of abnormalities in functional connectivity during resting state may be due to differences in analytic approach (pairwise network comparison vs. functional connectivity within networks and functional connectivity between specific brain regions), and/or age differences between samples across different investigations. Nevertheless, there is relatively consisntent evidence for functional connectivity abnormalities in URs (whether up or down) between brain regions involved in emotion processing and emotion regulation.

\subsubsection{Event-related potentials and the startle reflex}

Two studies have investigated event related potentials (ERPs) in URs (Katsanis et al., 1996; Pierson et al., 2000) (Table 3). One study found impairment in early as well as later stages of information processing, as reflected longer latency and reduced amplitude of N100 (an ERP measure of attention processes) and P300 (a measure of post-perceptual activity) (Pierson et al., 2000). The studies found no differences in simple visual stimuli processing as reflected by N200 and P200, which is in keeping with the findings in Katsanis et al.(1996). Finally, one study revealed abnormal threat processing in URs as indicated by reduced pre-pulse inhibition of the startle reflex (Giakoumaki et al., 2007).

\subsubsection{Interim summary}

Structural neuroimaging findings provide emerging evidence for increased amygdala and hippocampus volumes in young offspring of BD patients, but studies are scarce and the findings are inconsistent. Additionally, local reduction in white matter integrity is a relatively consistent finding in URs, although the locations of these changes vary across studies. Finally, a few studies found some evidence for abnormal electrophysiological measures and startle response, which point to abnormalities across different stages of information processing in URs.

\section{Discussion}

This systematic review identified and included ninety-seven studies the extant findings within 'cold' and 'hot' cognitive function and structural and resting-state neuroimagingn URs compared to HCs in order to identify the most promising endophenotypes for BD.

\section{1. 'Cold' and 'hot' cognition}

Overall, the most consistent and robust findings across the included literature were within the domains of 'cold' and 'hot' cognition. Studies on 'cold' cognition in healthy URs yielded consistent deficits within the domains of verbal learning and memory, sustained attention, and executive function. Functional MRI studies showed that the impairments in sustained attention and executive function were accompanied by aberrant neural activity in prefrontal, limbic, striatal and parietal regions. Studies on 'hot' cognition in URs showed consistent impairment of facial expression recognition, increased reactivity to emotional stimuli, impaired emotion regulation and increased interference of attentional resources by emotional stimuli. At the neural level, URs exhibited aberrant frontolimbic top-down regulation in to positive and negative stimuli and increased activation in reward processing areas, including the OFC. Fig. 2 provides a wide-angle lens synthesis of the most consistently replicated abnormalities in 'cold' and 'hot' cognition, as well as the underlying neurocircuitries.

Non-specific neurocognitive impairments within several aspects of 'cold' cognition are well-documented in schizophrenia and unipolar depression (UD) and in their URs (Lee et al., 2014; Rock et al., 2014; Snitz et al., 2006). As such, more pronounced general deficits, with regularly higher effect sizes, are found in relatives of schizophrenic (SC) compared to relatives of individuals with BD (Bora et al., 2009a; Snitz et al., 2006). 'Cold' cognition impairments in URs of patients with BD are therefore unlikely to represent a specific endophenotype to $\mathrm{BD}$, but rather appear to represent a broad, transdiagnostic marker of genetic vulnerability to neuropsychiatric illness in general. In contrast, changes in 'hot' cognition, particularly within reward and emotion processing and emotion regulation may represent a more specific endophenotype to $\mathrm{BD}$. In particular, aberrant emotion regulation and reward processing implicated in URs of patients with BD have not been shown in individuals at genetic risk for schizophrenia and may thus be a pertinent to mood disorders specifically (Meda et al., 2012). Studies of 'hot' cognition in individuals at genetic risk for UD show relatively consistent presence of negative bias (e.g. (Wolfensberger et al., 2008)) and attentional interference by negative stimuli (Feder et al., 2011; Joormann et al., 2007), which is accompanied by limbic hyperactivity and hypoactivity in ventral prefrontal regions, similar neural activity patterns in URs of patients with BD (for a review see Miskowiak and Carvalho, 2014). However, the latter group exhibits increased response to and deficient downregulation of emotional responses to both negative and positive stimuli. Failure to down-regulate emotional reactivity to positive stimuli may thus be an endophenotype that is specific to BD (Rive et al., 2015).

\subsection{Structural neuroimaging and resting-state findings}

Despite not finding any consistent patterns of grey matter differences, structural neuroimaging studies indicated local reduction in white matter integrity in regions involved in emotion processing and regulation. Resting-state fMRI studies provided consistent evidence for aberrant functional connectivity between frontal and limbic areas, although direction (i.e., up/down) of these abnormalities varied. In sum, neuroimaging findings showed some evidence for structural and resting state abnormalities in areas associated with emotion processing and regulation in URs, but findings were conflicting with regards to the direction and specific location of these changes. Studies reported are in general accordance with research on patients with BD showing functional abnormalities in neural circuits underlying emotion processing and regulation (see Strakowski et al., 2012 for a review). Specifically, aberrant frontallimbic activation, associated with emotion processing, has been exhibited in patients with BD and persists in periods of remission (Chen et al., 2011b; Phillips and Vieta, 2007).

\subsection{Limitations and implications}

This review provides a 'landscape' view of the studies in URs of BD patients within 'cold' and 'hot' cognition and neuroimaging. The studies included in the present review are cross-sectional, and thus embody a perspective that generally neglects the long-term development of the disorder (Frank et al., 2014). Longitudinal studies of URs are therefore critically needed to provide causal inferences, i.e. to indicate which of the candidate endophenotypes included in this review would predict the development of BD. In addition, the present systematic review included only original peer-reviewed articles. This is a possible methodological limitation because evidence suggests that studies with small sample sizes (which is particularly prevalent in the neuroimaging literature) may be prone to publication bias (Button et al., 2013). However, this systematic review included multiple procedures for identification of articles (i.e., database search and hand-searching of reference lists and tables of contents in the identified articles) thus limiting risk of bias following the PRISMA guidelines. Moreover, findings are difficult 


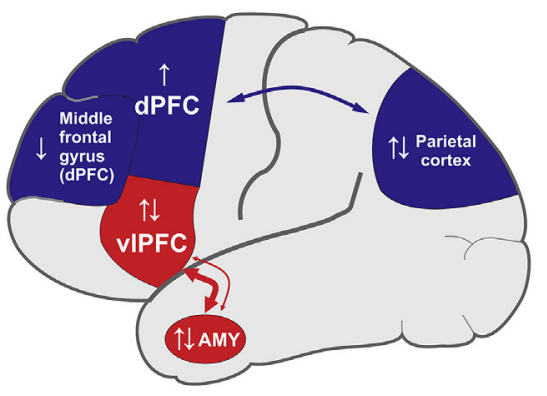

- Deficits in visual learning and memory (of faces) were associated with increased/decreased activity in hippocampal area, and decreased activity in frontal areas (ACC and middle frontal gyrus)

- Deficits in working memory were associated with increased activity in medial and dorsal PFC, and ACC

- Deficits in executive function were associated with increased activity in dorsal striatum (putamen and caudate) and aberrant parietal activity.

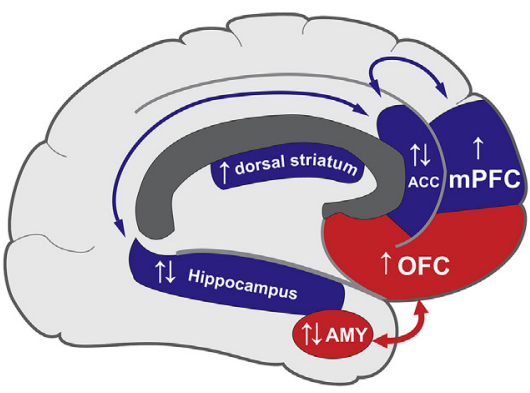

ABNORMALITIES IN HOT COGNITION

- Impaired emotion processing was associated with increased/decreased frontolimbic connectivity (most consistently VIPFC and amygdala)

- Impaired emotion regulation was associated with less PFC top-down regulation of amygdala, and increased positive frontolimbic (amgydala + OFC) connectivity.

Impaired reward processing was associated with increased OFC activity in response to reward.

Fig. 2. Summary of abnormalities in 'cold' and 'hot' cognition in unaffected relatives of bipolar patients.

to compare across studies partly due to different measures used, as well as heterogeneous and often small samples (i.e., relatives of BDI, BD-II, or both, without differentiation). In addition, although we aimed to exclude studies that provided data from overlapping samples (in these circumstances we considered the report that included the largest dataset), a residual overlapping of samples in different studies might have occurred. Finally, this review is limited by the lack of a guided quality assessment of studies. However, we extracted a wide range of information from included studies, and a critical appraisal of possible methodological limitations of included reports is provided in each section of this systematic review.

The present findings have important implications. The relatively consistent evidence for aberrant behavioural and neuronal measures of 'hot' cognition points to this domain as a putative endophenotype that could be specific for BD. This may inform our understanding of both pathophysiological mechanisms and treatment of BD. Specifically, increased sensitivity to reward and deficient emotion processing and regulation may amplify affect, thereby triggering a spiral into both manic and/or depressive states. As such, deficient prefrontal top-down regulation of limbic reactivity during positive emotion processing may represent a predisposition to (hypo)mania (Phillips and Swartz, 2014) and a endophenotype that could be implemented in the clinical assessments to improve future diagnostic discrimination between UD and BD (Cardoso de Almeida and Phillips, 2013; Chase et al., 2013; Pan et al., 2009). Moreover, the findings suggest that emotion dysregulation and aberrant reward processing is associated with genetic liability and may provide key targets for future preventive treatment strategies. Specifically, the individual patient's degree of reward sensitivity and difficulties with positive emotion regulation could potentially support the estimation of their Polarity Index (e.g. depressive vs. manic) (Carvalho et al., 2015) and thereby guide the choice of maintenance therapy.

The identification of endophenotypes that reflect underlying pathophysiological processes may confer risk or resilience toward future onset of $\mathrm{BD}$ in at-risk populations. This is vital for the development of novel treatments that specifically target these impairments in patients with BD and individuals at genetic risk. Indeed, preventive emotion-based treatments that aim to improve emotion resilience in at-risk individuals by enhancing their emo- tional knowledge, -regulation and - utilisation may delay the onset of the disorder or reduce the severity of pathological affective states (see Izard et al., 2008; van Zoonen et al., 2014). These mechanisms may aid the development of targeted psychotherapeutic interventions for the prevention of BD (Pfennig et al., 2014; Vallarino et al., 2015).

\section{Conclusion}

This review synthesises the extant literature on cognitive function, structural and functional neuroimaging measures in unaffected-first degree relatives of patients with BD compared to HCs. Our findings suggest that abnormalities within 'hot' cognition, specifically aberrant emotion processing and - regulation and reward processing represent the most promising specific endophenotypic marker for BD. Future research is warranted to elucidate the role of 'hot' cognition and associated neural abnormalities as an endophenotype for BD to help improve diagnostic accuracy and develop targeted treatments in at-risk individuals. In addition, the development of novel analytic strategies (e.g., machine learning approaches) may aid the identification of multi-modal endophenotypes of BD that integrate illness-related changes in neurocognitive and neuroimaging measures (Passos et al., 2016).

\section{Conflict of interest}

KWM reports having received consultancy fees from Lundbeck and Allergan. MV discloses consultancy fees from Lundbeck, Servier and Astra Zeneca within the last three years. LVK reports having been a consultant for Lundbeck and AstraZeneca within the last 3 years. HLK, IM, JZP, BRC, CAK, and AFC report no conflicts of interest.

\section{Acknowledgements}

The Lundbeck Foundation has provided half of KWM's postdoctorate salary at the Psychiatric Center Copenhagen for her to do full-time research (2012-2015). AFC is the recipient of a research fellowship award from the Conselho de Desenvolvimento Científico e Tecnológico (CNPq, Level II, Brazil). CAK is the recipient of a research fellowship award from CAPES (Brazil). 


\section{Appendix A. Supplementary data}

Supplementary data associated with this article can be found, in the online version, at http://dx.doi.org/10.1016/j.neubiorev.2016. 12.011 .

\section{References}

Adleman, N.E., Yi, J.Y., Deveney, C.M., Guyer, A.E., Leibenluft, E., Brotman, M.A., 2014. Increased intrasubject variability in response time in unaffected preschoolers at familial risk for bipolar disorder. Psychiatry Res. 219, 687-689.

Almeida, J.R.C., Phillips, M.L., 2013. Distinguishing between unipolar depression and bipolar depression: current and future clinical and neuroimaging perspectives. Biol. Psychiatry 73, 111-118.

Antila, M., Tuulio-Henriksson, A., Kieseppa, T., Eerola, M., Partonen, T., Lonnqvist, J., 2007. Cognitive functioning in patients with familial bipolar I disorder and their unaffected relatives. Psychol. Med. 37, 679-687.

Arnone, D., Cavanagh, J., Gerber, D., Lawrie, S.M., Ebmeier, K.P., McIntosh, A.M., 2009. Magnetic resonance imaging studies in bipolar disorder and schizophrenia: meta-analysis. Br. J. Psychiatry 195, 194-201.

Arslan, F.C., Tiryaki, A., Ozkorumak, E., 2014. A comparison of euthymic bipolar patients with unaffected first-degree relatives and healthy controls in terms of neuropsychological functions. Int. J. Psychiatry Clin. Pract. 18, 208-214.

Arts, B., Jabben, N., Krabbendam, L., van Os, J., 2008. Meta-analyses of cognitive functioning in euthymic bipolar patients and their first-degree relatives. Psychol. Med. 38, 771-785.

Association, A.P., 2013. Diagnostic and Statistical Manual of Mental Disorders $\left(\mathrm{DSM}-5^{\oplus}\right)$. American Psychiatric Pub.

Balanza-Martinez, V., Rubio, C., Selva-Vera, G., Martinez-Aran, A., Sanchez-Moreno, J., Salazar-Fraile, J., Vieta, E., Tabares-Seisdedos, R., 2008. Neurocognitive endophenotypes (endophenocognitypes) from studies of relatives of bipolar disorder subjects: a systematic review. Neurosci. Biobehav. Rev. 32, $1426-1438$

Bauer, I.E., Sanches, M., Suchting, R., Green, C.E., El Fangary, N.M., Zunta-Soares, G.B., Soares, J.C., 2014. Amygdala enlargement in unaffected offspring of bipolar parents. J. Psychiatr. Res. 59, 200-205.

Besnier, N., Richard, F., Zendjidjian, X., Kaladjian, A., Mazzola-Pomietto, P., Adida, M., Azorin, J.M., 2009. Stroop and emotional Stroop interference in unaffected relatives of patients with schizophrenic and bipolar disorders: distinct markers of vulnerability? World J. Biol. Psychiatry 10, 809-818.

Bora, E., Pantelis, C., 2015. Meta-analysis of social cognition in attention-deficit/hyperactivity disorder (ADHD): Comparison with healthy controls and autistic spectrum disorder. Psychol. Med. 1, 1-18.

Bora, E., Vahip, S., Akdeniz, F., Ilerisoy, H., Aldemir, E., Alkan, M., 2008. Executive and verbal working memory dysfunction in first-degree relatives of patients with bipolar disorder. Psychiatry Res. 161, 318-324.

Bora, E., Yucel, M., Pantelis, C., 2009a. Cognitive endophenotypes of bipolar disorder: a meta-analysis of neuropsychological deficits in euthymic patients and their first-degree relatives. J. Affect. Disord. 113, 1-20.

Bora, E., Yucel, M., Pantelis, C., 2009b. Cognitive endophenotypes of bipolar disorder: a meta-analysis of neuropsychological deficits in euthymic patients and their first-degree relatives. J. Affect. Disord. 113, 1-20.

Bourne, C., Aydemir, O., Balanza-Martinez, V., Bora, E., Brissos, S., Cavanagh, J.T., Clark, L., Cubukcuoglu, Z., Dias, V.V., Dittmann, S., Ferrier, I.N., Fleck, D.E., Frangou, S., Gallagher, P., Jones, L., Kieseppa, T., Martinez-Aran, A., Melle, I., Moore, P.B., Mur, M., Pfennig, A., Raust, A., Senturk, V., Simonsen, C., Smith, D.J., Bio, D.S., Soeiro-de-Souza, M.G., Stoddart, S.D., Sundet, K., Szoke, A., Thompson, J.M., Torrent, C., Zalla, T., Craddock, N., Andreassen, O.A., Leboyer, M., Vieta, E., Bauer, M., Worhunsky, P.D., Tzagarakis, C., Rogers, R.D., Geddes, J.R., Goodwin, G.M., 2013. Neuropsychological testing of cognitive impairment in euthymic bipolar disorder: an individual patient data meta-analysis. Acta Psychiatr. Scand. 128, 149-162.

Brand, J.G., Goldberg, T.E., Gunawardane, N., Gopin, C.B., Powers, R.L., Malhotra, A.K., Burdick, K.E., 2012. Emotional bias in unaffected siblings of patients with bipolar I disorder. J. Affect. Disord. 136, 1053-1058.

Brotman, M.A., Guyer, A.E., Lawson, E.S., Horsey, S.E., Rich, B.A., Dickstein, D.P., Pine, D.S., Leibenluft, E., 2008a. Facial emotion labeling deficits in children and adolescents at risk for bipolar disorder. Am. J. Psychiatry 165, 385-389.

Brotman, M.A., Skup, M., Rich, B.A., Blair, K.S., Pine, D.S., Blair, J.R., Leibenluft, E., 2008b. Risk for bipolar disorder is associated with face-processing deficits across emotions. J. Am. Acad. Child Adolesc. Psychiatry 47, 1455-1461.

Brotman, M.A., Rooney, M.H., Skup, M., Pine, D.S., Leibenluft, E., 2009. Increased intrasubject variability in response time in youths with bipolar disorder and at-risk family members. J. Am. Acad. Child Adolesc. Psychiatry 48, 628-635.

Brotman, M.A., Deveney, C.M., Thomas, L.A., Hinton, K., Yi, J., Pine, D.S., Leibenluft, E., 2014. Parametric modulation of neural activity during face emotion processing in unaffected youth at familial risk for bipolar disorder. Neuropsychopharmacology 38, S132-S133.

Button, K.S., Ioannidis, J.P.A., Mokrysz, C., Nosek, B.A., Flint, J., Robinson, E.S.J., Munafo, M.R., 2013. Power failure: why small sample size undermines the reliability of neuroscience. Nat. Rev. Neurosci. 14, 365-376.

Cardoso de Almeida, J.R., Phillips, M.L., 2013. Distinguishing between unipolar depression and bipolar depression: current and future clinical and neuroimaging perspectives. Biol. Psychiatry 73, 111-118.
Carvalho, A.F., Quevedo, J., McIntyre, R.S., Soeiro-de-Souza, M.G., Fountoulakis, K.N., Berk, M., Hyphantis, T.N., Vieta, E., 2015. Treatment implications of predominant polarity and the polarity index: a comprehensive review. Int. J. Neuropsychopharmacol. 18.

Cha, D.S., De Michele, F., Soczynska, J.K., Woldeyohannes, H.O., Kaidanovich-Beilin, O., Carvalho, A.F., Malhi, G.S., Patel, H., Sim, K., Brietzke, E., Mansur, R., Dunlop, K.A., Alsuwaidan, M., Baskaran, A., Fagiolini, A., Reznikov, R., Kudlow, P.A., McIntyre, R.S., 2014. The putative impact of metabolic health on default mode network activity and functional connectivity in neuropsychiatric disorders. CNS Neurol. Disord. Drug Targets 13, 1750-1758.

Chang, B.P., Lenzenweger, M.F., 2005. Somatosensory processing and schizophrenia liability: proprioception, exteroceptive sensitivity, and graphesthesia performance in the biological relatives of schizophrenia patients. J. Abnorm. Psychol. 114, 85-95.

Chase, H.W., Nusslock, R., Almeida, J.R., Forbes, E.E., LaBarbara, E.J., Phillips, M.L., 2013. Dissociable patterns of abnormal frontal cortical activation during anticipation of an uncertain reward or loss in bipolar versus major depression. Bipolar Disord. 15, 839-854.

Chen, C.-H., Suckling, J., Lennox, B.R., Ooi, C., Bullmore, E.T., 2011a. A quantitative meta-analysis of fMRI studies in bipolar disorder. Bipolar Disord. 13, 1-15.

Chen, C.H., Suckling, J., Lennox, B.R., Ooi, C., Bullmore, E.T., 2011b. A quantitative meta-analysis of fMRI studies in bipolar disorder. Bipolar Disord. 13, 1-15.

Christodoulou, T., Messinis, L., Papathanasopoulos, P., Frangou, S., 2012a. Dissociable and common deficits in inhibitory control in schizophrenia and bipolar disorder. Eur. Arch. Psychiatry Clin. Neurosci. 262, 125-130.

Christodoulou, T., Messinis, L., Papathanasopoulos, P., Frangou, S., 2012b. The impact of familial risk for schizophrenia or bipolar disorder on cognitive control during episodic memory retrieval. Psychiatry Res. 197, 212-216.

Civil Arslan, F., Tiryaki, A., Ozkorumak, E., 2014. A comparison of euthymic bipolar patients with unaffected first-degree relatives and healthy controls in terms of neuropsychological functions. Int. J. Psychiatry Clin. Pract. 18, 208-214.

Costafreda, S.G., Fu, C.H., Picchioni, M., Kane, F., McDonald, C., Prata, D.P., Kalidindi, S., Walshe, M., Curtis, V., Bramon, E., Kravariti, E., Marshall, N., Toulopoulou, T., Barker, G.J., David, A.S., Brammer, M.J., Murray, R.M., McGuire, P.K., 2009. Increased inferior frontal activation during word generation: a marker of genetic risk for schizophrenia but not bipolar disorder? Hum. Brain Mapp. 30, 3287-3298

Daban, C., Mathieu, F., Raust, A., Cochet, B., Scott, J., Etain, B., Leboyer, M., Bellivier, F., 2012. Is processing speed a valid cognitive endophenotype for bipolar disorder? J. Affect. Disord. 139, 98-101.

Deveci, E., Ozan, E., Kirpinar, I., Oral, M., Daloglu, A.G., Aydin, N., Ozturk, A., 2013. Neurocognitive functioning in young high-risk offspring having a parent with bipolar I disorder. Turk. J. Med. Sci. 43, 110-117.

Deveney, C.M., Connolly, M.E., Jenkins, S.E., Kim, P., Fromm, S.J., Brotman, M.A., Pine, D.S., Leibenluft, E., 2012. Striatal dysfunction during failed motor inhibition in children at risk for bipolar disorder. Prog. Neuro-Psychopharmacol. Biol. Psychiatry 38, 127-133.

Dima, D., Roberts, R.E., Frangou, S., 2016. Connectomic markers of disease expression, genetic risk and resilience in bipolar disorder. Transl. Psychiatry 6 (no pagination)

Doyle, A.E., Wozniak, J., Wilens, T.E., Henin, A., Seidman, L.J., Petty, C., Fried, R., Gross, L.M., Faraone, S.V., Biederman, J., 2009. Neurocognitive impairment in unaffected siblings of youth with bipolar disorder. Psychol. Med. 39, 1253-1263

Eker, C., Simsek, F., Yilmazer, E.E., Kitis, O., Cinar, C., Eker, O.D., Coburn, K., Gonul, A.S., 2014. Brain regions associated with risk and resistance for bipolar I disorder: a voxel-based MRI study of patients with bipolar disorder and their healthy siblings. Bipolar Disord. 16, 249-261.

Erk, S., Meyer-Lindenberg, A., Schmierer, P., Mohnke, S., Grimm, O., Garbusow, M., Haddad, L., Poehland, L., Muhleisen, T.W., Witt, S.H., Tost, H., Kirsch, P. Romanczuk-Seiferth, N., Schott, B.H., Cichon, S., Nothen, M.M., Rietschel, M., Heinz, A., Walter, H., 2014. Hippocampal and frontolimbic function as intermediate phenotype for psychosis: evidence from healthy relatives and a common risk variant in cacna1c. Biol. Psychiatry 76, 466-475.

Erol, A., Kosger, F., Putgul, G., Ersoy, B., 2014. Ventral prefrontal executive function impairment as a potential endophenotypic marker for bipolar disorder. Nord. J. Psychiatry 68, 18-23.

Feder, A., Skipper, J., Blair, J.R., Buchholz, K., Mathew, S.J., Schwarz, M., Doucette, J.T., Alonso, A., Collins, K.A., Neumeister, A., Charney, D.S., 2011. Tryptophan depletion and emotional processing in healthy volunteers at high risk for depression. Biol. Psychiatry 69, 804-807.

Frangou, S., Haldane, M., Roddy, D., Kumari, V., 2005. Evidence for deficit in tasks of ventral, but not dorsal, prefrontal executive function as an endophenotypic marker for bipolar disorder. Biol. Psychiatry 58, 838-839.

Frangou, S., 2012. Brain structural and functional correlates of resilience to bipolar disorder. Front. Hum. Neurosci., 1-10.

Frantom, L.V., Allen, D.N., Cross, C.L., 2008. Neurocognitive endophenotypes for bipolar disorder. Bipolar Disord. 10, 387-399.

Frazier, J.A., Breeze, J.L., Papadimitriou, G., Kennedy, D.N., Hodge, S.M., Moore, C.M., Howard, J.D., Rohan, M.P., Caviness, V.S., Makris, N., 2007. White matter abnormalities in children with and at risk for bipolar disorder. Bipolar Disord. 9, 799-809.

Gatt, J.M., Burton, K.L., Williams, L.M., Schofield, P.R., 2015. Specific and common genes implicated across major mental disorders: a review of meta-analysis studies. J. Psychiatr. Res. 60, 1-13. 
Gershon, E.S., Goldin, L.R., 1986. Clinical methods in psychiatric genetics: i. Robustness of genetic marker investigative strategies. Acta Psychiatr. Scand. $74,113-118$

Giakoumaki, S.G., Roussos, P., Rogdaki, M., Karli, C., Bitsios, P., Frangou, S., 2007. Evidence of disrupted prepulse inhibition in unaffected siblings of bipolar disorder patients. Biol. Psychiatry 62, 1418-1422.

Giakoumaki, S.G., Bitsios, P., Frangou, S., Roussos, P., Aasen, I., Galea, A., Kumari, V. 2010. Low baseline startle and deficient affective startle modulation in remitted bipolar disorder patients and their unaffected siblings. Psychophysiology 47, 659-668

Glahn, D.C., Almasy, L., Barguil, M., Hare, E., Peralta, J.M., Kent Jr., J.W., Dassori, A., Contreras, J., Pacheco, A., Lanzagorta, N., Nicolini, H., Raventos, H., Escamilla, M.A., 2010. Neurocognitive endophenotypes for bipolar disorder identified in multiplex multigenerational families. Arch. Gen. Psychiatry 67, 168-177.

Goes, F.S., 2016. Genetics of bipolar disorder: recent update and future directions. Psychiat. Clinics North Am. 39, 139-155.

Goodwin, F.K., Jamison, K.R., 2007. Manic-depressive Illness: Bipolar Disorders and Recurrent Depression, 2 ed. Oxford University Press, New York, NY.

Gottesman, I.I., Gould, T.D., 2003. The endophenotype concept in psychiatry etymology and strategic intentions. Am. J. Psychiatry 160, 636-645.

Grande, I., Berk, M., Birmaher, B., Vieta, E., 2016. Bipolar disorder. Lancet (Lond. Engl.) 387, 1561-1572.

Green, M.J., Lino, B.J., Hwang, E.J., Sparks, A., James, C., Mitchell, P.B., 2011. Cognitive regulation of emotion in bipolar I disorder and unaffected biological relatives. Acta Psychiatr. Scand. 124, 307-316.

Gunde, E., Novak, T., Kopecek, M., Schmidt, M., Propper, L., Stopkova, P., Hoschl, C., Duffy, A., Alda, M., Hajek, T., 2011. White matter hyperintensities in affected and unaffected late teenage and early adulthood offspring of bipolar parents: two-center high-risk study. J. Psychiatr. Res. 45, 76-82.

Hıdıroğlu, C., Torres, I.J., Er, A., Işık, G., Yalın, N., Yatham, L.N., Ceylan, D., Özerdem, A., 2015. Response inhibition and interference control in patients with bipolar disorder and first-degree relatives. Bipolar Disord. 17, 781-794.

Haas, B.W., Omura, K., Constable, R.T., Canli, T., 2007. Emotional conflict and neuroticism: personality-dependent activation in the amygdala and subgenual anterior cingulate. Behav. Neurosci. 121, 249-256.

Hajek, T., Cullis, J., Novak, T., Kopecek, M., Blagdon, R., Propper, L., Stopkova, P., Duffy, A., Hoschl, C., Uher, R., Paus, T., Young, L.T., Alda, M., 2013. Brain structural signature of familial predisposition for bipolar disorder: replicable evidence for involvement of the right inferior frontal gyrus. Biol. Psychiatry 73 . $144-152$

Hajek, T., Cooke, C., Kopecek, M., Novak, T., Hoschl, C., Alda, M., 2015. Using structural MRI to identify individuals at genetic risk for bipolar disorders: a 2-cohort, machine learning study. J. Psychiatry Neurosci. 1, 8872147.

Hasler, G., Drevets, W.C., Gould, T.D., Gottesman, I.I., Manji, H.K., 2006. Toward constructing an endophenotype strategy for bipolar disorders. Biol. Psychiatry 60, 93-105.

Heissler, J., Kanske, P., Schönfelder, S., Wessa, M., 2014. Inefficiency of emotion regulation as vulnerability marker for bipolar disorder: evidence from healthy individuals with hypomanic personality. J. Affect. Disord. 152, 83-90.

Hidiroglu, C., Demirci Esen, O., Tunca, Z., Neslihan Garz Yalcin, S., Lombardo, L. Glahn, D.C., Ozerdem, A., 2013. Can risk-taking be an endophenotype for bipolar disorder? A study on patients with bipolar disorder type I and their first-degree relatives. J. Int. Neuropsychol. Soc. 19, 474-482.

Hozer, F., Houenou, J., 2016. Can neuroimaging disentangle bipolar disorder? J. Affect. Disord. 195, 199-214.

Izard, C., Stark, K., Trentacosta, C., Schultz, D., 2008. Beyond emotion regulation: emotion utilization and adaptive functioning. Child Dev. Perspect. 2, 156-163.

Joormann, J., Talbot, L., Gotlib, I.H., 2007. Biased processing of emotional information in girls at risk for depression. J. Abnorm. Psychol. 116, 135-143.

Juselius, S., Kieseppa, T., Kaprio, J., Lonnqvist, J., Tuulio-Henriksson, A., 2009. Executive functioning in twins with bipolar I disorder and healthy co-twins. Arch. Clin. Neuropsychol. 24, 599-606.

Kanske, P., Schonfelder, S., Forneck, J., Wessa, M., 2015. Impaired regulation of emotion: neural correlates of reappraisal and distraction in bipolar disorder and unaffected relatives. Transl. Psychiatry 5, e497.

Katsanis, J., Iacono, W.G., Beiser, M., 1996. Visual event-related potentials in first-episode psychotic patients and their relatives. Psychophysiology 33, 207-217

Kempton, M.J., Geddes, J.R., Ettinger, U., Williams, S.C.R., Grasby, P.M., 2008. Meta-analysis, database, and meta-regression of 98 structural imaging studies in bipolar disorder. Arch. Gen. Psychiatry 65, 1017-1032.

Keri, S., Kelemen, O., Benedek, G., Janka, Z., 2001. Different trait markers for schizophrenia and bipolar disorder: a neurocognitive approach. Psychol. Med. 31, 915-922.

Kerner, B., 2015. Toward a deeper understanding of the genetics of bipolar disorder. Front. Psychiatry 6, 105

Kieseppä, T., Partonen, T., Haukka, J., Kaprio, J., Lönnqvist, J., 2004. High concordance of bipolar I disorder in a nationwide sample of twins. Am. J. Psychiatry 161, 1814-1821

Kim, P., Jenkins, S.E., Connolly, M.E., Deveney, C.M., Fromm, S.J., Brotman, M.A. Nelson, E.E., Pine, D.S., Leibenluft, E., 2012. Neural correlates of cognitive flexibility in children at risk for bipolar disorder. J. Psychiatr. Res. 46, 22-30.

Kim, D., Kim, J., Koo, T., Yun, H., Won, S., 2015. Shared and distinct neurocognitive endophenotypes of schizophrenia and psychotic bipolar disorder. Clin. Psychopharmacol. Neurosci. 13, 94-102.
Kosger, F., Essizoglu, A., Baltacioglu, M., Ulkgun, N., Yenilmez, C., 2015. Executive function in parents of patients with familial versus sporadic bipolar disorder. Compr. Psychiatry 61, 36-41.

Kulkarni, S., Jain, S., Janardhan Reddy, Y.C., Kumar, K.J., Kandavel, T., 2010. Impairment of verbal learning and memory and executive function in unaffected siblings of probands with bipolar disorder. Bipolar Disord. 12, 647-656.

Ladouceur, C.D., Almeida, J.R., Birmaher, B., Axelson, D.A., Nau, S., Kalas, C., Monk, K., Kupfer, D.J., Phillips, M.L., 2008. Subcortical gray matter volume abnormalities in healthy bipolar offspring: potential neuroanatomical risk marker for bipolar disorder? J. Am. Acad. Child Adolesc. Psychiatry 47, 532-539.

Ladouceur, C.D., Diwadkar, V.A., White, R., Bass, J., Birmaher, B., Axelson, D.A. Phillips, M.L., 2013. Fronto-limbic function in unaffected offspring at familial risk for bipolar disorder during an emotional working memory paradigm. Dev. Cognit. Neurosci. 5, 185-196.

Leboyer, M., Bellivier, F., Nosten-Bertrand, M., Jouvent, R., Pauls, D., Mallet, J., 1998. Psychiatric genetics: search for phenotypes. Trends Neurosi. 21, 102-105.

Lee, S.H., Sung, K., Lee, K.S., Moon, E., Kim, C.G., 2014. Mismatch negativity is a stronger indicator of functional outcomes than neurocognition or theory of mind in patients with schizophrenia. Prog. Neuro-Psychopharmacol. Biol. Psychiatry 48, 213-219.

Li, C.T., Tu, P.C., Hsieh, J.C., Lee, H.C., Bai, Y.M., Tsai, C.F., Wang, S.J., Hsu, J.W., Huang, K.L., Hong, C.J., 2015. Functional dysconnection in the prefrontal-amygdala circuitry in unaffected siblings of patients with bipolar I disorder. Bipolar Disord. 17, 626-635.

Linke, J., King, A.V., Rietschel, M., Strohmaier, J., Hennerici, M., Gass, A., Meyer-Lindenberg, A., Wessa, M., 2012. Increased medial orbitofrontal and amygdala activation: evidence for a systems-level endophenotype of bipolar disorder. Am. J. Psychiatry 169, 316-325

Linke, J., King, A.V., Poupon, C., Hennerici, M.G., Gass, A., Wessa, M., 2013. Impaired anatomical connectivity and related executive functions: differentiating vulnerability and disease marker in bipolar disorder. Biol. Psychiatry 74, 908-916.

Lui, S., Yao, L., Xiao, Y., Keedy, S., Reilly, J., Keefe, R., Tamminga, C., Keshavan, M., Pearlson, G., Gong, Q., 2015. Resting-state brain function in schizophrenia and psychotic bipolar probands and their first-degree relatives. Psychol. Med. 45, 97-108.

Mathias de Almeida, K., Nery, F.G., Moreno, R.A., Gorenstein, C., Lafer, B., 2013. A sib-pair analysis of impulsivity in bipolar disorder type I. Compr. Psychiatry 54, 1148-1152.

Matsuo, K., Kopecek, M., Nicoletti, M., Hatch, J., Watanabe, Y., Nery, F. Zunta-Soares, G., Soares, J., 2012. New structural brain imaging endophenotype in bipolar disorder. Mol. Psychiatry 17, 412-420.

Maziade, M., Rouleau, N., Gingras, N., Boutin, P., Paradis, M.-E., Jomphe, V., Boutin, J., Létourneau, K., Gilbert, E., Lefebvre, A.-A., 2009. Shared neurocognitive dysfunctions in young offspring at extreme risk for schizophrenia or bipolar disorder in eastern quebec multigenerational families. Schizophr. Bull. 35, 919-930.

McCarroll, S.A., Feng, G., Hyman, S.E., 2014. Genome-scale neurogenetics: methodology and meaning. Nat. Neurosci. 17, 756-763.

McDonald, C., Zanelli, J., Rabe-Hesketh, S., Ellison-Wright, I., Sham, P., Kalidindi, S., Murray, R.M., Kennedy, N., 2004. Meta-analysis of magnetic resonance imaging brain morphometry studies in bipolar disorder. Biol. Psychiatry 56, 411-417.

McGuffin, P., Rijsdijk, F., Andrew, M., Sham, P., Katz, R., Cardno, A., 2003. The heritability of bipolar affective disorder and the genetic relationship to unipolar depression. Arch. Gen. Psychiatry 60, 497-502.

Meda, S.A., Gill, A., Stevens, M.C., Lorenzoni, R.P., Glahn, D.C., Calhoun, V.D., Sweeney, J.A., Tamminga, C.A., Keshavan, M.S., Thaker, G., Pearlson, G.D., 2012. Differences in resting-state functional magnetic resonance imaging functional network connectivity between schizophrenia and psychotic bipolar probands and their unaffected first-degree relatives. Biol. Psychiatry 71, 881-889.

Miskowiak, K.W., Carvalho, A.F., 2014. Hot cognition in major depressive disorder: a systematic review. CNS Neurol. Disord. Drug Targets 13, 1787-1803.

Moher, D., Liberati, A., Tetzlaff, J., Altman, D.G., 2009. Preferred reporting items for systematic reviews and meta-analyses: the PRISMA statement. BMJ (Clin. Res. Ed.) 339 , b2535

Nehra, R., Grover, S., Sharma, S., Sharma, A., Sarkar, S., 2014. Neuro-cognitive functioning in unaffected siblings of patients with bipolar disorder: comparison with bipolar patients and healthy controls. Indian J. Psychiatry 56, 283.

Nurnberger Jr., J.I., Koller, D.L., Jung, J., Edenberg, H.J., Foroud, T., Guella, I., Vawter, M.P., Kelsoe, J.R., 2014. Identification of pathways for bipolar disorder: a meta-analysis. JAMA Psychiatry 71, 657-664.

Olsavsky, A.K., Brotman, M.A., Rutenberg, J.G., Muhrer, E.J., Deveney, C.M., Fromm, S.J., Towbin, K., Pine, D.S., Leibenluft, E., 2012. Amygdala hyperactivation during face emotion processing in unaffected youth at risk for bipolar disorder. J. Am. Acad. Child Adolesc. Psychiatry 51, 294-303.

Organization, W.H., 1992. The ICD-10 Classification of Mental and Behavioural Disorders: Clinical Descriptions and Diagnostic Guidelines. World Health Organization, Geneva.

Pan, L., Keener, M.T., Hassel, S., Phillips, M.L., 2009. Functional neuroimaging studies of bipolar disorder: examining the wide clinical spectrum in the search for disease endophenotypes. Int. Rev. Psychiatry (Abingdon, England) 21, 368-379.

Passos, I.C., Mwangi, B., Kapczinski, F., 2016. Big data analytics and machine learning: 2015 and beyond. Lancet. Psychiatry 3, 13-15. 
Patino, L.R., Adler, C.M., Mills, N.P., Strakowski, S.M., Fleck, D.E., Welge, J.A., DelBello, M.P., 2013. Conflict monitoring and adaptation in individuals at familial risk for developing bipolar disorder. Bipolar Disord. 15, 264-271.

Pattanayak, R.D., Sagar, R., Mehta, M., 2012. Neurocognition in unaffected first-degree relatives of patients with bipolar disorder type I from India: a potential vulnerability marker? SAGE Open, 2.

Pavlickova, H., Turnbull, O., Bentall, R.P., 2014. Cognitive vulnerability to bipolar disorder in offspring of parents with bipolar disorder. Br. J. Clin. Psychol. 53, 386-401.

Pfennig, A., Leopold, K., Bechdolf, A., Correll, C.U., Holtmann, M., Lambert, M., Marx, C., Meyer, T.D., Pfeiffer, S., Reif, A., Rottmann-Wolf, M., Schmitt, N.M., Stamm, T., Juckel, G., Bauer, M., 2014. Early specific cognitive-behavioural psychotherapy in subjects at high risk for bipolar disorders: study protocol for a randomised controlled trial. Trials 15, 161.

Phillips, M.L., Kupfer, D.J., 2013. Bipolar disorder diagnosis: challanges and future directions. Lancet 381, 1663-1671.

Phillips, M.L., Swartz, H.A., 2014. A critical appraisal of neuroimaging studies of bipolar disorder: toward a new conceptualization of underlying neural circuitry and roadmap for future research. Am. J. Psychiatry 171, 829-843.

Phillips, M.L., Vieta, E., 2007. Identifying functional neuroimaging biomarkers of bipolar disorder: towards DSM-V. Schizophr. Bull. 33, 893-904.

Phillips, M.L., Ladouceur, C.D., Drevets, W.C., 2008. A neural model of voluntary and automatic emotion regulation: implications for understanding the pathophysiology and neurodevelopment of bipolar disorder. Mol. Psychiatry 13 (829), 833-857.

Pierson, A., Jouvent, R., Quintin, P., Perez-Diaz, F., Leboyer, M., 2000. Information processing deficits in relatives of manic depressive patients. Psychol. Med. 30 $545-555$.

Raichle, M.E., Snyder, A.Z., 2007. A default mode of brain function: a brief history of an evolving idea. Neuroimage 37, 1083-1090.

Rive, M.M., Mocking, R.J., Koeter, M.W., van Wingen, G., de Wit, S.J., van den Heuvel, O.A., Veltman, D.J., Ruhe, H.G., Schene, A.H., 2015. State-dependent differences in emotion regulation between unmedicated bipolar disorder and major depressive disorder. JAMA Psychiatry 72, 687-696.

Robinson, L.J., Thompson, J.M., Gallagher, P., Goswami, U., Young, A.H., Ferrier, I.N., Moore, P.B., 2006. A meta-analysis of cognitive deficits in euthymic patients with bipolar disorder. J. Affect. Disord. 93, 105-115.

Rock, P.L., Roiser, J.P., Riedel, W.J., Blackwell, A.D., 2014. Cognitive impairment in depression: a systematic review and meta-analysis. Psychol. Med. 44 2029-2040

Rodriguez-Jimenez, R., Bagney, A., Garcia-Navarro, C., Aparicio, A.I., Lopez-Anton, R., Moreno-Ortega, M., Jimenez-Arriero, M.A., Santos, J.L., Lobo, A., Kern, R.S., Green, M.F., Nuechterlein, K.H., Palomo, T., 2012. The MATRICS consensus cognitive battery (MCCB): co-norming and standardization in Spain. Schizophr. Res. 134, 279-284.

Roiser, J.P., Sahakian, B.J., 2013. Hot and cold cognition in depression. CNS Spectr. $18,139-149$.

Sandoval, H., Soares, J.C., Mwangi, B., Asonye, S., Alvarado, L.A., Zavala, J., Ramirez, M.E., Sanches, M., Enge, L.R., Escamilla, M.A., 2016. Confirmation of MRI anatomical measurements as endophenotypic markers for bipolar disorder in a new sample from the NIMH Genetics of Bipolar Disorder in Latino Populations study. Psychiatry Res. 247, 34-41.

Saricicek, A., Yalin, N., Hidiroglu, C., Cavusoglu, B., Tas, C., Ceylan, D., Zorlu, N., Ada, E., Tunca, Z., Ozerdem, A., 2015. Neuroanatomical correlates of genetic risk for bipolar disorder: a voxel-based morphometry study in bipolar type I patients and healthy first degree relatives. J. Affect. Disord. 186, 110-118.

Seidel, E.-M., Habel, U., Finkelmeyer, A., Hasmann, A., Dobmeier, M., Derntl, B., 2012. Risk or resilience?: Empathic abilities in patients with bipolar disorders and their first-degree relatives. J. Psychiatr. Res. 46, 382-388.

Sepede, G., De Berardis, D., Campanella, D., Perrucci, M.G., Ferretti, A., Serroni, N., Moschetta, F.S., Del Gratta, C., Salerno, R.M., Ferro, F.M., Di Giannantonio, M., Onofrj, M., Romani, G.L., Gambi, F., 2012. Impaired sustained attention in euthymic bipolar disorder patients and non-affected relatives: an fMRI study. Bipolar Disord. 14, 764-779.

Sepede, G., De Berardis, D., Campanella, D., Perrucci, M.G., Ferretti, A., Salerno, R.M., Di Giannantonio, M., Romani, G.L., Gambi, F., 2015. Neural correlates of negative emotion processing in bipolar disorder. Prog. Neuro-Psychopharmacol. Biol. Psychiatry 60, 1-10.

Singh, M.K., Chang, K.D., Kelley, R.G., Saggar, M., Reiss, A.L., Gotlib, I.H., 2014a. Early signs of anomalous neural functional connectivity in healthy offspring of parents with bipolar disorder. Bipolar Disord. 16, 678-689.

Singh, M.K., Kelley, R.G., Howe, M.E., Reiss, A.L., Gotlib, I.H., Chang, K.D., 2014b. Reward processing in healthy offspring of parents with bipolar disorder. JAMA Psychiatry 71, 1148-1156.

Snitz, B.E., Macdonald 3rd, A.W., Carter, C.S., 2006. Cognitive deficits in unaffected first-degree relatives of schizophrenia patients: a meta-analytic review of putative endophenotypes. Schizophr. Bull. 32, 179-194.

Sobczak, S., Riedel, W.J., Booij, I., Aan Het Rot, M., Deutz, N.E., Honig, A., 2002. Cognition following acute tryptophan depletion: difference between first-degree relatives of bipolar disorder patients and matched healthy control volunteers. Psychol. Med. 32, 503-515.

Sobczak, S., Honig, A., Schmitt, J.A., Riedel, W.J., 2003. Pronounced cognitive deficits following an intravenous L-tryptophan challenge in first-degree relatives of bipolar patients compared to healthy controls. Neuropsychopharmacology 28 , 711-719.

Strakowski, S.M., Delbello, M.P., Adler, C.M., 2005. The functional neuroanatomy of bipolar disorder: a review of neuroimaging findings. Mol. Psychiatry 10, 105-116

Strakowski, S.M., Adler, C.M., Almeida, J., Altshuler, L.L., Blumberg, H.P., Chang, K.D., DelBello, M.P., Frangou, S., McIntosh, A., Phillips, M.L., Sussman, J.E., Townsend, J.D., 2012. The functional neuroanatomy of bipolar disorder: a consensus model. Bipolar Disord. 14, 313-325.

Surguladze, S.A., Marshall, N., Schulze, K., Hall, M.H., Walshe, M., Bramon, E., Phillips, M.L., Murray, R.M., McDonald, C., 2010. Exaggerated neural response to emotional faces in patients with bipolar disorder and their first-degree relatives. Neuroimage 53, 58-64.

Szoke, A., Schurhoff, F., Golmard, J.L., Alter, C., Roy, I., Meary, A., Etain, B., Bellivier, F., Leboyer, M., 2006a. Familial resemblance for executive functions in families of schizophrenic and bipolar patients. Psychiatry Res. 144, 131-138.

Szoke, A., Schurhoff, F., Meary, A., Mathieu, F., Chevalier, F., Trandafir, A., Alter, C., Roy, I., Bellivier, F., Leboyer, M., 2006b. Lack of influence of COMT and NET genes variants on executive functions in schizophrenic and bipolar patients, their first-degree relatives and controls. Am. J. Med. Genet. 141b, 504-512.

Teixeira, A.M.A., Kleinman, A., Zanetti, M., Jackowski, M., Duran, F., Pereira, F., Lafer, B., Busatto, G.F., Caetano, S.C., 2014. Preserved white matter in unmedicated pediatric bipolar disorder. Neurosci. Lett. 579, 41-45.

Thermenos, H.W., Makris, N., Whitfield-Gabrieli, S., Brown, A.B., Giuliano, A.J., Lee, E.H., Faraone, S.V., Tsuang, M.T., Seidman, L.J., 2011. A functional MRI study of working memory in adolescents and young adults at genetic risk for bipolar disorder: preliminary findings. Bipolar Disord. 13, 272-286.

Tighe, S.K., Reading, S.A., Rivkin, P., Caffo, B., Schweizer, B., Pearlson, G., Potash, J.B., DePaulo, J., Bassett, S.S., 2012. Total white matter hyperintensity volume in bipolar disorder patients and their healthy relatives. Bipolar Disord. 14, $888-893$.

Torres, I.J., Boudreau, V.G., Yatham, L.N., 2007. Neuropsychological functioning in euthymic bipolar disorder: A meta-analysis. Acta Psychiatr. Scand. 116, 17-26.

Townsend, J.D., Torrisi, S.J., Lieberman, M.D., Sugar, C.A., Bookheimer, S.Y., Altshuler, L.L., 2013. Frontal-amygdala connectivity alterations during emotion downregulation in bipolar I disorder. Biol. Psychiatry 73, 127-135.

Trivedi, J.K., Goel, D., Dhyani, M., Sharma, S., Singh, A.P., Sinha, P.K., Tandon, R., 2008. Neurocognition in first-degree healthy relatives (siblings) of bipolar affective disorder patients. Psychiatry Clin. Neurosci. 62, 190-196.

Tseng, W.-L., Bones, B.L., Kayser, R.R., Olsavsky, A.K., Fromm, S.J., Pine, D.S., Leibenluft, E., Brotman, M.A., 2015. An fMRI study of emotional face encoding in youth at risk for bipolar disorder. Eur. Psychiatry 30, 94-98.

Uher, R., 2014. Gene-environment interactions in severe mental illness. Front. Psychiatry 5, 48.

Vallarino, M., Henry, C., Etain, B., Gehue, L.J., Macneil, C., Scott, E.M., Barbato, A., Conus, P., Hlastala, S.A., Fristad, M., Miklowitz, D.J., Scott, J., 2015. An evidence map of psychosocial interventions for the earliest stages of bipolar disorder. Lancet. Psychiatry 2, 548-563.

Van Rheenen, T.E., Rossell, S.L., 2014. An empirical evaluation of the MATRICS Consensus Cognitive Battery in bipolar disorder. Bipolar Disord. 16, 318-325.

van Zoonen, K., Buntrock, C., Ebert, D.D., Smit, F., Reynolds 3rd, C.F., Beekman, A.T. Cuijpers, P., 2014. Preventing the onset of major depressive disorder: a meta-analytic review of psychological interventions. Int. J. Epidemiol. 43, 318-329.

Vargas, C., López-Jaramillo, C., Vieta, E., 2013. A systematic literature review of resting state network-functional MRI in bipolar disorder. J. Affect. Disord. 150, 727-735.

Versace, A., Ladouceur, C.D., Romero, S., Birmaher, B., Axelson, D.A., Kupfer, D.J., Phillips, M.L., 2010. Altered development of white matter in youth at high familial risk for bipolar disorder: a diffusion tensor imaging study. J. Am. Acad. Child Adolesc. Psychiatry 49 (1249-1259), e1241.

Wessa, M., Kollmann, B., Linke, J., Schonfelder, S., Kanske, P., 2015. Increased impulsivity as a vulnerability marker for bipolar disorder: evidence from self-report and experimental measures in two high-risk populations. J. Affect. Disord. 178, 18-24.

Wolfensberger, S.P., Veltman, D.J., Hoogendijk, W.J., Boomsma, D.I., de Geus, E.J., 2008. Amygdala responses to emotional faces in twins discordant or concordant for the risk for anxiety and depression. Neuroimage 41, 544-552.

Wu, M.J., Mwangi, B., Bauer, I.E., Passos, I.C., Sanches, M., Zunta-Soares, G.B., Meyer, T.D., Hasan, K.M., Soares, J.C., 2016. Identification and individualized prediction of clinical phenotypes in bipolar disorders using neurocognitive data, neuroimaging scans and machine learning. Neuroimage.

Zalla, T., Joyce, C., Szoke, A., Schurhoff, F., Pillon, B., Komano, O., Perez-Diaz, F., Bellivier, F., Alter, C., Dubois, B., Rouillon, F., Houde, O., Leboyer, M., 2004. Executive dysfunctions as potential markers of familial vulnerability to bipolar disorder and schizophrenia. Psychiatry Res. 121, 207-217. 